US Army Corps

of Engineers

Waterways Experiment

Station

\title{
Investigation of the Constitutive Model Used in Nonlinear, Incremental Structural Analyses
}

by Barry D. Fehl, Sharon B. Garner

Approved For Public Release; Distribution Is Unlimited 
The contents of this report are not to be used for advertising, publication, or promotional purposes. Citation of trade names does not constitute an official endorsement or approval of the use of such commercial products.

The findings of this report are not to be construed as an official Department of the Army position, unless so designated by other authorized documents. 


\section{Investigation of the Constitution Model Used in Nonlinear, Incremental Structural Analysis}

by Barry D. Fehl, Sharon B. Gamer

U.S. Army Corps of Engineers

Waterways Experiment Station

3909 Halls Ferry Road

Vicksburg, MS 39180-6199

Final report

Approved for public release; distribution is unlimited 


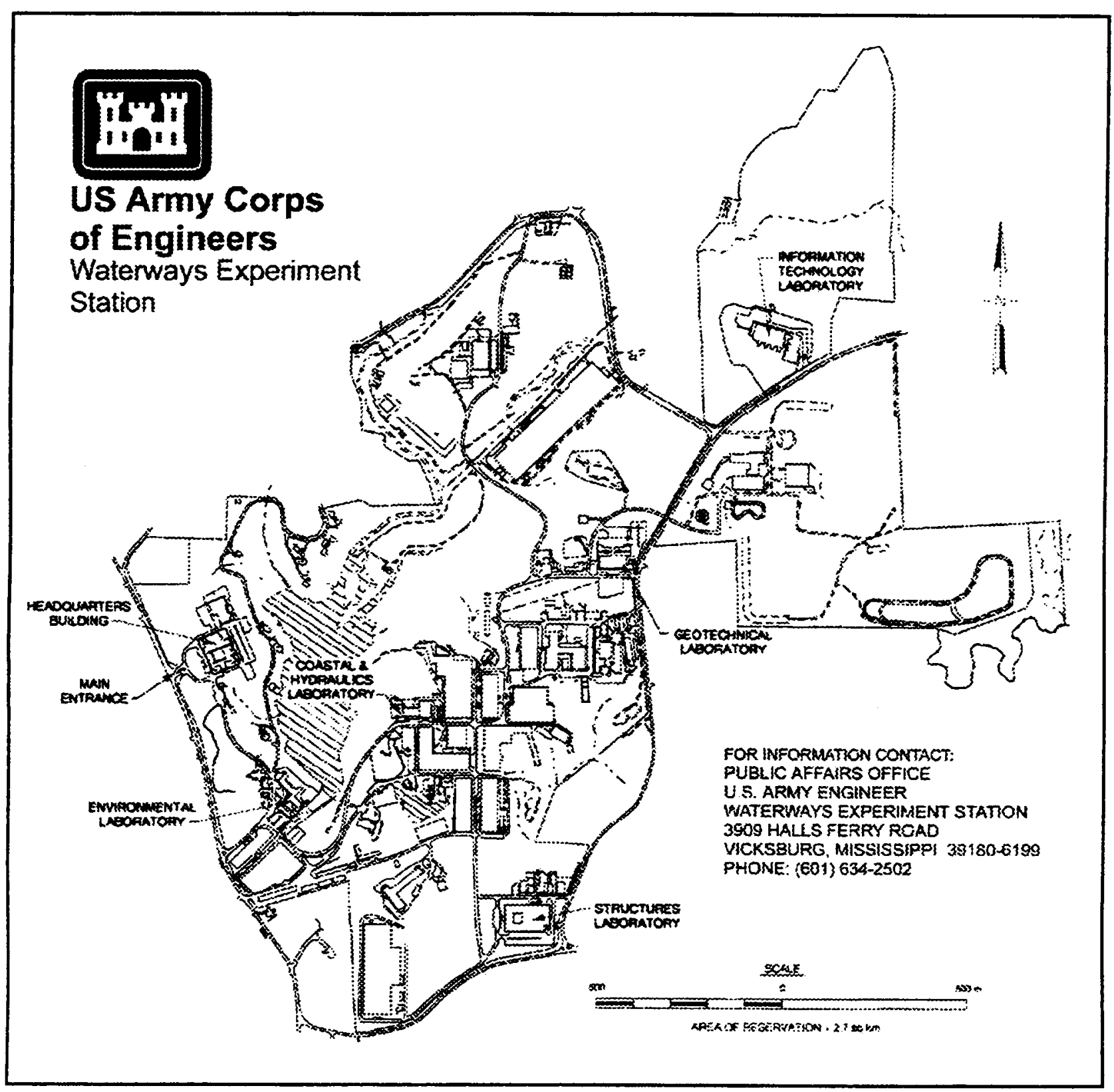

\section{Waterways Experiment Station Cataloging-in-Publication Data}

Fehl, Barry D., 1957-

Investigation of the constitutive model used in nonlinear incremental structural analysis /

by Barry D. Fehl, Sharon B. Gamer ; prepared for U.S. Army Corps of Engineers.

54 p. : ill. ; $28 \mathrm{~cm}$. - (Technical report ; ITL-98-1)

Includes bibliographic references.

1. Structural analysis (Engineering -- Testing. 2. Nonlinear mechanics -- Testing. 3. Concrete -- Testing. I. Garner, Sharon B. II. United States. Army. Corps of Engineers. III. U.S. Army Engineer Waterways Experiment Station. IV. Information Technology Laboratory (U.S. Army Engineer Waterways Experiment Station) V. Title. VI. Series: Technical report (U.S. Army Engineer Waterways Experiment Station) ; ITL-98-1.

TA7 W34 no.ITL-98-1 


\section{Contents}

Preface $\ldots \ldots \ldots \ldots \ldots \ldots \ldots \ldots \ldots \ldots \ldots \ldots \ldots \ldots \ldots \ldots \ldots$ vi

Conversion Factors $\ldots \ldots \ldots \ldots \ldots \ldots \ldots \ldots \ldots \ldots \ldots \ldots \ldots \ldots \ldots$ vii

1 -Introduction $\ldots \ldots \ldots \ldots \ldots \ldots \ldots \ldots \ldots \ldots \ldots \ldots \ldots \ldots$

2-Multiaxial Creep $\ldots \ldots \ldots \ldots \ldots \ldots \ldots \ldots \ldots \ldots \ldots \ldots \ldots$

Background ........................ 4

Description of Verification Tests $\ldots \ldots \ldots \ldots \ldots \ldots \ldots \ldots \ldots \ldots \ldots 5$

Description of FE Model $\ldots \ldots \ldots \ldots \ldots \ldots \ldots \ldots \ldots \ldots \ldots, 5$

FE Analyses Results $\ldots \ldots \ldots \ldots \ldots \ldots \ldots \ldots \ldots \ldots \ldots \ldots \ldots . \ldots$

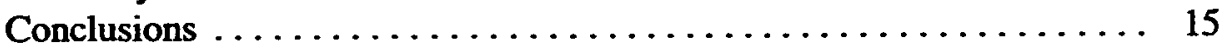

$3-$ Creep Strain Recovery $\ldots \ldots \ldots \ldots \ldots \ldots \ldots \ldots \ldots \ldots \ldots \ldots$

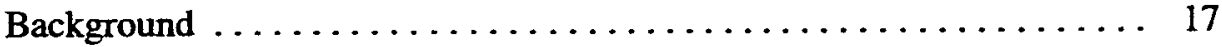

FE Modeling of Creep and Creep Recovery $\ldots \ldots \ldots \ldots \ldots \ldots \ldots$

Analyses Results ......................... 19

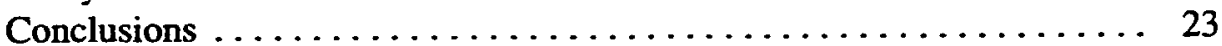

4 Slow Load Beam Test $\ldots \ldots \ldots \ldots \ldots \ldots \ldots \ldots \ldots \ldots \ldots$

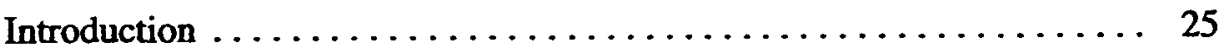

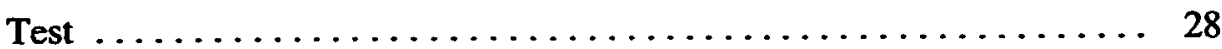

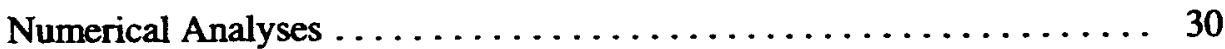

Conclusion ............................. 36

5 -Rapid Load Beam Test $\ldots \ldots \ldots \ldots \ldots \ldots \ldots \ldots \ldots \ldots \ldots \ldots \ldots$

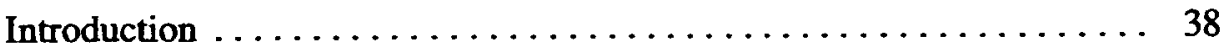

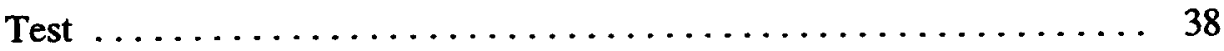

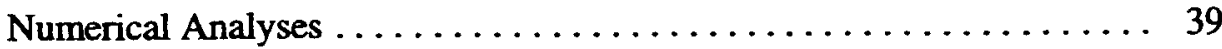

Conclusions .......................... 41

$6-$ Conclusions and Recommendations $\ldots \ldots \ldots \ldots \ldots \ldots \ldots \ldots \ldots \ldots \ldots$

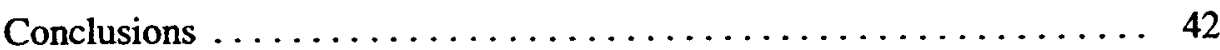

Recommendations .......................... 43

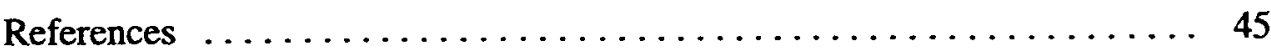

SF 298 
Figure 1. FE grid $\ldots \ldots \ldots \ldots \ldots \ldots \ldots \ldots \ldots \ldots \ldots \ldots \ldots \ldots \ldots$

Figure 2. Test and predicted strains, E-39 $\ldots \ldots \ldots \ldots \ldots \ldots \ldots$

Figure 3. Test and predicted strains, $B-7 \ldots \ldots \ldots \ldots \ldots$

Figure 4. Test and predicted strains, 600 -psi biaxial loading $\ldots \ldots \ldots \ldots 9$

Figure 5. Test and predicted strains, 3,600-psi biaxial load $\ldots \ldots \ldots \ldots 9$

Figure 6. Test and predicted strains, 2,400-psi axial pressure, 600-psi radial pressure $\ldots \ldots \ldots \ldots \ldots \ldots \ldots \ldots \ldots \ldots \ldots \ldots$

Figure 7. Test and predicted strains, 3,600-psi axial pressure, 1,200-psi radial pressure $\ldots \ldots \ldots \ldots \ldots \ldots \ldots \ldots \ldots \ldots \ldots \ldots \ldots$

Figure 8. Test and predicted strains, 600-psi hydrostatic loading . . . . . 11

Figure 9. Test and predicted strains, 2,400-psi hydrostatic loading $\ldots \ldots 12$

Figure 10. Test and predicted creep strains, 3,600-psi hydrostatic

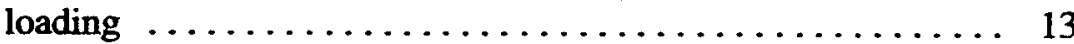

Figure 11. Test and predicted creep strains, 1,200-psi hydrostatic loading with loss of radial load after 90 minutes . . . . . . . . . . . 13

Figure 12. Test and predicted strains for 1,200-psi axial loading, 2,400 -psi radial loading $\ldots \ldots \ldots \ldots \ldots \ldots \ldots \ldots \ldots \ldots \ldots$

Figure 13. Test and predicted creep strains for 600 -psi axial loading, 3,600 -psi radial loading . . . . . . . . . . . . . . . . 14

Figure 14. Test and predicted strains, 2,400-psi axial creep test, McDonald main modulus concrete $\ldots \ldots \ldots \ldots \ldots \ldots \ldots \ldots$

Figure 15. Test and predicted creep strains, triaxial test, McDonald main modulus concrete $\ldots \ldots \ldots \ldots \ldots \ldots \ldots \ldots \ldots \ldots, 16$

Figure 16. 7-day creep test with loading lost on day $8 \ldots \ldots \ldots \ldots \ldots$

Figure 17. Typical specific creep curves, aging and non-aging materials . . . 19

Figure 18. Uniaxial and biaxial creep recovery $\ldots \ldots \ldots \ldots \ldots \ldots$

Figure 19. Hydrostatic test and predicted creep recovery $\ldots \ldots \ldots \ldots \ldots 21$

Figure 20. Test and predicted creep recovery, triaxial tests $\ldots \ldots \ldots \ldots 22$

Figure 21. Test and predicted creep and creep recovery strains, E-39 . . . 23

Figure 22. Geometry and loading of beam used in slow load test ...... 25

Figure 23. Stress-strain interaction diagram used as cracking threshold in constitutive model $\ldots \ldots \ldots \ldots \ldots \ldots \ldots \ldots \ldots \ldots$

Figure 24. Cracking potential generation for a specific cracking failure surface 
Figure 25. Comparison of recommended loading of slow load beam and

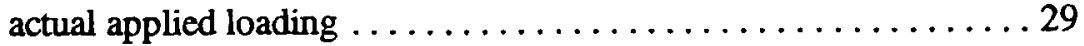

Figure 26. Meshes used to analyze the slow load beam test $\ldots \ldots \ldots \ldots$

Figure 27. Strain time-histories of measured strains in actual test and predicted strains from numerical analyses using constant value of 117.2 in./in. for $\epsilon_{f} \ldots \ldots \ldots \ldots \ldots \ldots \ldots \ldots \ldots \ldots \ldots \ldots \ldots$

Figure 28. Strain time-histories of measured strains in actual test and predicted strains from numerical analyses using adjusted

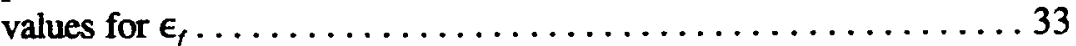

Figure 29. Strain histories of measured strains in actual test and predicted cracking strains from numerical analyses using

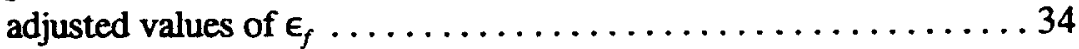

Figure 30. Strain time-histories of measured strains in actual test and predicted strains from numerical analyses using adjusted values for $\epsilon_{f}$ and no initial temperature of $70^{\circ} \mathrm{F} \ldots \ldots .35$

Figure 31. Strain time-histories of measured strains in actual test and predicted strains from numerical analyses using adjusted values for $\epsilon_{f}$ and no intial temperature of $70{ }^{\circ} \mathrm{F}$ but with shrinkage strains included $\ldots \ldots \ldots \ldots \ldots \ldots \ldots \ldots \ldots \ldots$

Figure 32. Measured strain versus predicted strain for rapid load test

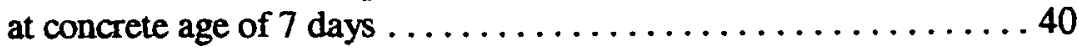

Figure 33. Measured strain versus predicted strain for rapid load test at concrete age of 109 days 


\section{Preface}

This report was written to document work performed in the area of verification of the constitutive model used in the nonlinear, incremental structural analysis methodology. The work was sponsored under funds provided to the U.S. Army Engineer Waterways Experiment Station (WES) by the Engineering Division of Headquarters, U.S. Army Corps of Engineers, as part of the Computer-Aided Analysis of Massive Concrete Structures work unit of the Structural Engineering Research and Development Program.

The report was written by Mr. Barry D. Fehl, Computer-Aided Engineering Division (CAED), Information Technology Laboratory (TTL), WES, and Ms. Sharon B. Garner, Structural Mechanics Division (SMD), Structures Laboratory (SL), WES. The work was coordinated in ITL by Mr. Fehl, under the general supervision of Mr. H. Wayne Jones, Chief, CAED, and Dr. N. Radhakrishnan, Director, ITL. Dr. Robert Hall, Chief, Structural Analysis Group, SL, was the Program Manager for the Structural Engineering and Concrete R\&D Program. Dr. Reed Mosher was Chief, SMD; Dr. Bryant Mather was Director, SL.

At the time of publication of this report, Director of WES was Dr. Robert W. Whalin. Commander was COL Robin R. Cababa, EN. 


\section{Conversion Factors, Non-SI to SI Units of Measurement}

Non-SI units of measurement used in this report can be converted to SI units as follows:

\begin{tabular}{|l|l|l|}
\hline Multuply & By & To Obtaln \\
\hline degrees Fahrenheit & 519 & degrees Celsius $\alpha$ kelvins' \\
\hline feet & 0.3048 & meters \\
\hline inches & 25.4 & millimeters \\
\hline pounds (force) per square inch & 0.006894757 & megapascals \\
\hline pounds (mass) & 0.4535924 & kilograms \\
\hline $\begin{array}{l}\text { ' To obtain Celsius }(C) \text { temperature reading from Fahrenheit }(F) \text { readings, use tho following } \\
\text { formula: } C=(5 / 9)(F-32) \text {. To cbtain Kelvin }(K) \text { readings, use } K=(5 / 9)(F-32)+273.15 .\end{array}$ \\
\hline
\end{tabular}




\section{Introduction}

The nonlinear, incremental structural analysis (NISA) procedure was developed in the mid-1980s concurrently under the Cracking of Concrete work unit of the Repair, Evaluation, Maintenance, and Rehabilitation (REMR) Program and the Melvin Price Locks and Dam Project. The NISA procedure was developed to update a methodology used since the late 1960 s to evaluate mass concrete structures. While the programs used for evaluating mass concrete structures which had been developed by Sandu, William, and Raphael (1967) and Wilson (1968) had been used successfully, the process required updating to take advantage of the increased computational capacity of computers and improved finite element methods.

The efforts resulted in the development of a procedure to be used for the evaluation of massive concrete structures. Following its use on the Melvin Price Project, NISA studies were performed on Lock and Dam Nos. 4 and 5 on the Red River, Olmsted Locks on the Ohio River, and the McAlpine Lock Replacement Project, also on the Ohio River. Formal guidance for performing a NISA was also developed. An Engineer Technical Letter (ETL 1110-2-324), "Special Design Provisions for Massive Concrete Structures" (U.S. Army Corps of Engineers (USACE) 1990), was the original guidance published and was replaced by ETL 1110-2-365 (USACE 1994) "Nonlinear, Incremental Structural Analysis of Massive Concrete Structures" to update the guidance to correspond to the current state of the art.

The concurrent effort in development of the NISA methodology resulted in many procedural issues in performing a NISA to be resolved during the Melvin Price Project effort, while the efforts of the Cracking of Concrete work unit concentrated on the development of a constitutive model which would capture the age-dependent characteristics of creep, shrinkage, and the modulus of elasticity of concrete. In addition, a smeared crack model was to be included in the constitutive model to allow for crack prediction in structures. The general purpose finite element package, ABAQUS, was chosen for performing NISA studies in part because user supplied subroutines could be used for constitutive relationships. After a decision was made regarding the finite element code, the constitutive model for young concrete was developed by ANATECH Corporation utilizing their smeared cracking and creep model used for evaluations of concrete containments and underground waste storage tanks. This constitutive model is contained in the proprietary software ANACAP-U (ANATECH 1992b). 
In the analysis procedure developed by the U.S. Army Engineer Waterways Experiment Station (WES) and ANATECH, the equations in the constitutive model were calibrated for each project based on results of laboratory tests of the actual materials expected to be used at the project site. A review of the equations within the constitutive model and the method of calibrating the model is contained in WES Technical Report ITL-95-8, "Constitutive Modeling of Concrete for Massive Concrete Structures, A Simplified Overview" (Truman and Fehl 1995). This report details the parameters required to model elasticity, creep, and shrinkage in an aging model and how to modify the input parameters of the constitutive model so that they approximate the results obtained from the test data.

Despite the fact that the NISA process was established based on past experiences in performing finite element analyses of massive concrete structures and that the constitutive model was being calibrated to actual test results, questions have been raised concerning the validity of the NISA process. Many of these questions are concerned with the validity of using laboratory tests to capture behavior that is occurring within a massive concrete structure. There were also concerns about the validity of using a linear viscoelastic creep model based on uniaxial test data and applying it to all states of stress.

Due to the concerns regarding the NISA procedure and the constitutive model, an effort was undertaken as part of the Structural Engineering and Concrete Program work unit "Computer-Aided Analysis of Massive Concrete Structures" to validate the NISA procedure. This effort was initiated during a discussion between a group of engineers and scientists with extensive experience in concrete testing and numerical modeling of concrete. The discussions centered on how the NISA methodology, particularly the constitutive model, could be validated and how this could be accomplished using existing data from the laboratory.

The result of the discussions led to a proposed plan for validating the constitutive model used in NISA. The following validation efforts were proposed:

a. Perform analyses of slow and rapid load beam tests performed for the McAlpine Lock Replacement Project and compare analyses results with test data.

b. Perform creep, shrinkage, and modulus tests using mixture proportions and materials from the McAlpine project to check the capability of repeating the results of the original set of tests.

c. Compare model predictions of creep recovery with existing test data to check the capability of the numerical model to produce an adequate representation of unloading or creep recovery.

d. Perform a biaxial creep test and a corresponding finite element analysis to determine if the use of creep curves based on uniaxial tests provides accurate predictions for other states of stress. 
e. Test a representative mass concrete placement in the laboratory with controlled boundary conditions. Analysis of the slab should be performed prior to the performance of the test to establish the test parameters. Multiple lifts are necessary and cracking should occur.

It was agreed that if it could be shown that the constitutive model performed satisfactorily in the above tests, then the model could be used with a high level of confidence. It was also recognized that the results of the proposed plan may show that adjustment to the constitutive model is necessary.

This report addresses items $a, c$, and $d$ of the above plan. Numerical studies were performed, and the results of the numerical studies are compared with the actual data. The slow load and rapid load beam results are compared with results obtained during testing performed during the McAlpine Lock Replacement Project. The creep recovery and biaxial numerical studies are compared against existing test data rather than performing a new test. This varies from the original proposal. However, since a literature search provided reliable test data on biaxial, hydrostatic, and triaxial states of stress, the study was expanded to include these loading conditions, and existing data were used for the comparison.

Items $b$ and $e$ will be addressed in future work. 


\section{Multiaxial Creep}

\section{Background}

Creep is the time-dependent deformation of concrete subjected to a sustained loading. Creep strain is the total strain of a loaded specimen minus the elastic strain that occurs upon loading and the shrinkage and thermally induced strains determined from control specimens.

The majority of creep tests are uniaxial compressive tests conducted in accordance with American Society for Testing and Materials (ASTM) Standard C 512-87 (1992a), which requires that the maximum test load be no more than 40 percent of the compressive strength of the concrete at the age of loading. At levels of stress below this value, creep is assumed to be a linear function of stress. At higher levels of stress, creep can be highly nonlinear. The ANACAP-U model uses this assumption of creep linearity to predict creep strain under all states of stress. Since maximum stress in mass concrete is limited by the tensile capacity of the concrete and is normally significantly less than 40 percent of the compressive strength, this assumption of linearity is applicable for uniaxial loadings, both compressive and tensile. However, there is much less data available on multiaxial creep testing, and available test data are often inconsistent.

Multiaxial creep tests cited in American Concrete Institute (ACI) publication SP-27 (1970) resulted in conflicting conclusions about the effects of state of stress on creep. However, many of the differences were attributed to differences in environmental conditions, with drying being the most influential factor. This is a logical conclusion since creep is commonly aknowleged to be heavily dependent on moisture migration. The ANACAP aging viscoelastic model was designed for use on mass concrete, in which drying only occurs near the surface and the majority of the concrete is isolated from moisture loss. To accurately model the moisture conditions of mass concrete, only tests on sealed specimens were used to verify ANACAP multiaxial predictions. 


\section{Description of Verification Tests}

Creep test results used in the multiaxial creep verification were reported by York, Kennedy, and Perry (1970) and McDonald (1973). In these tests, uniaxial, biaxial, and triaxial creep tests were conducted on 6 -in. ${ }^{1}$ by 18 -in. or 6 -in. by 16-in. cylinders, and unloaded companion cylinders were monitored for shrinkage strains. Test cylinders were cured using two different processes: as-cast or air dried. As-cast cylinders were kept in a fog room for 1 to 2 days, then sealed against moisture loss with two coats of epoxy and enclosed in copper sleeves. Two temperatures at loading were also considered in each series of tests: $75^{\circ} \mathrm{F}$ and $150^{\circ} \mathrm{F}$ in the tests by York, Kennedy, and Perry (1970), $73^{\circ} \mathrm{F}$ and $150^{\circ} \mathrm{F}$ in the tests by McDonald (1973). Cylinders were loaded at 90 days after casting, and loadings were maintained for 1 year. Since conditions in the interior of a mass concrete structure could be expected to be similar to those of the as-cast 73 or $75^{\circ} \mathrm{F}$ cylinders at 90 days after placement, only the tests that were performed under as-cast cuuring conditions were modeled in the finite element analyses.

In the tests, axial loads were applied by a hydraulic ram and monitored by a load cell. Radial loads were applied by hydraulic pressure through oil contained by a steel pressure jacket. A control system automatically regulated pressures to within 5 percent of the design pressures except in the event of a power failure or a major oil leak.

Compressive strength, elastic modulus $E$, and Poisson's ratio at loading, and design loadings for the as-cast test cylinders are listed in Table 1. All tests designated by a single letter and a number (for example, B-7) are by York, Kennedy, and Perry (1970).

\section{Description of FE Model}

The tests were simulated using the finite element (FE) program ABAQUS and the aging viscoelastic material model ANACAP-U developed by ANATECH Corporation. A one-quarter symmetric section of a cylinder was modeled using eight-node axisymmetric elements, and the appropriate uniform pressures were applied at the top and outer side of the grid. The grid used in the FE analyses is shown in Figure 1.

Test E-39, a 600-psi uniaxial test, was used to calibrate the creep curve in ANACAP-U. Due to lack of data, no attempt was made to model the aging modulus. Although changes in modulus are used to translate the creep curve in time (ANATECH 1992a), the increase in modulus after 90 days is relatively small. Since these test simulations were begun at an initial age of 90 days, errors due to using a modulus curve based on data from other concretes should be

\footnotetext{
${ }^{1}$ A table of factors for converting non-SI units of measurement to SI units of measurement is found on page vi.
} 


\begin{tabular}{|c|c|c|c|c|c|}
\hline \multirow[b]{2}{*}{ Test } & \multirow{2}{*}{$\begin{array}{l}\text { 90-Day } \\
\text { Compressive } \\
\text { Strength, psi }\end{array}$} & \multicolumn{2}{|c|}{ Elastic Parameters } & \multicolumn{2}{|c|}{ Design Pressure, psi } \\
\hline & & E, pol $\times 10^{\circ}$ & $\begin{array}{l}\text { Polsson's } \\
\text { Ratio }\end{array}$ & Axial & Radial \\
\hline $8-7$ & 6,110 & 5.66 & 0.243 & 2,400 & 0 \\
\hline$B-41$ & 6,110 & 7.14 & 0.239 & 1,200 & 2,400 \\
\hline$C-16$ & 6,430 & 2.04 & 0.409 & 1,200 & 1,200 \\
\hline$c-23$ & 6,430 & 6.54 & 0.244 & 2,400 & 600 \\
\hline D-26 & 6,500 & 5.91 & 0.273 & 3,600 & 1,200 \\
\hline$D-31$ & 6,500 & 3.11 & 0.358 & 3,600 & 3,600 \\
\hline E-5 & 7,290 & 2.78 & 0.381 & 600 & 600 \\
\hline$E-39$ & 7,290 & 6.04 & 0.293 & 600 & 0 \\
\hline$F-9$ & 7,410 & 12.55 & 0.017 & 2,400 & 2,400 \\
\hline$F-13$ & 7,410 & 6.13 & 0.264 & 0 & 600 \\
\hline G-35 & 6.460 & 4.91 & 0.248 & 600 & 3,600 \\
\hline $1+22$ & 6,330 & 4.51 & 0.209 & 0 & 3,600 \\
\hline McDonald Axial & 7,660 & 5.50 & & 2,400 & 0 \\
\hline $\begin{array}{l}\text { McDonald } \\
\text { Triaxial }\end{array}$ & 7,660 & 5.50 & & 2,400 & 600 \\
\hline
\end{tabular}

small. The fit to the test E-39 strains was based upon the McAlpine exterior mixture creep and modulus curves (Bombich, Neeley, and Garner.) ${ }^{1}$ The creep curve was then modified by means of the creep factor until predicted creep strains closely matched test strains. Predicted and test axial strains are shown in Figure 2. The 2,400-psi axial test, B-7, was modeled to verify this fit. For this analysis, predicted axial creep strains were 94.4 percent of test strains on day 360. Predicted and test axial creep strains are shown in Figure 3. These axial test simulations produced satisfactory results, and the calibration was then used in the simulation of all tests by York, Kennedy, and Perry (1970).

1 Bombich, A. A., Neeley, B. D., and Garner, S. B. "Concrete mixture selection and characterization study McAlpine locks replacement, Ohio River," (technical report in preparation), U.S. Army Engineer Waterways Experiment Station, Vicksburg, MS. 


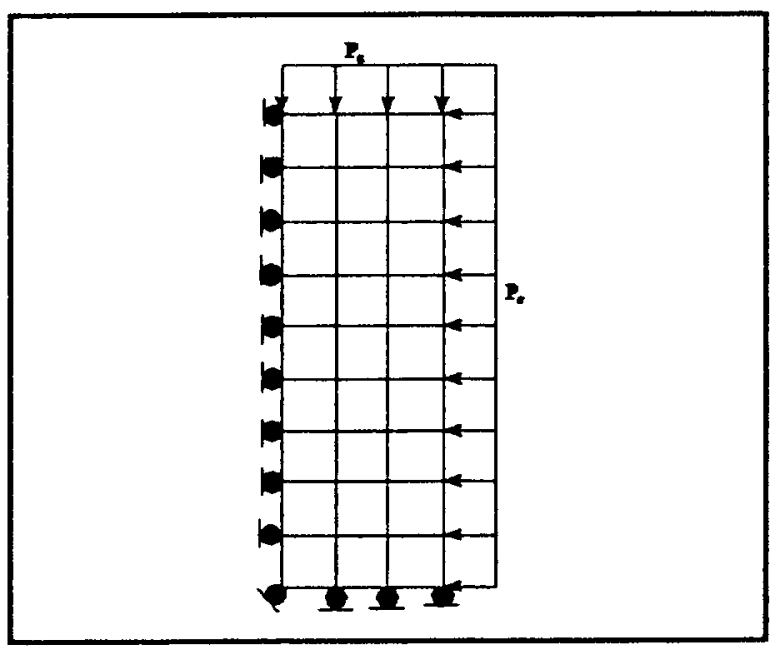

Figure 1. FE grid

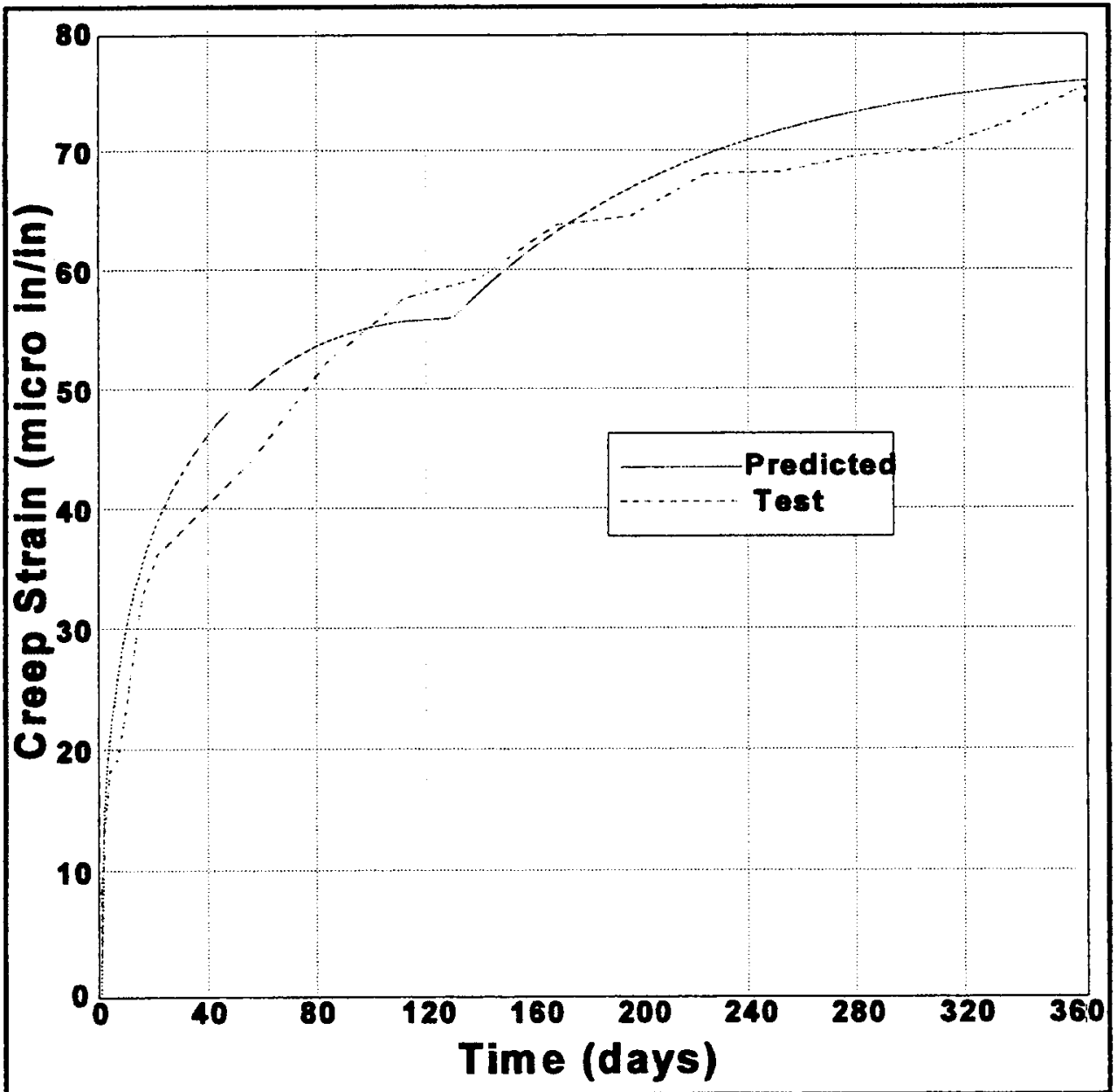

Figure 2. Test and predicted strains, E-39 


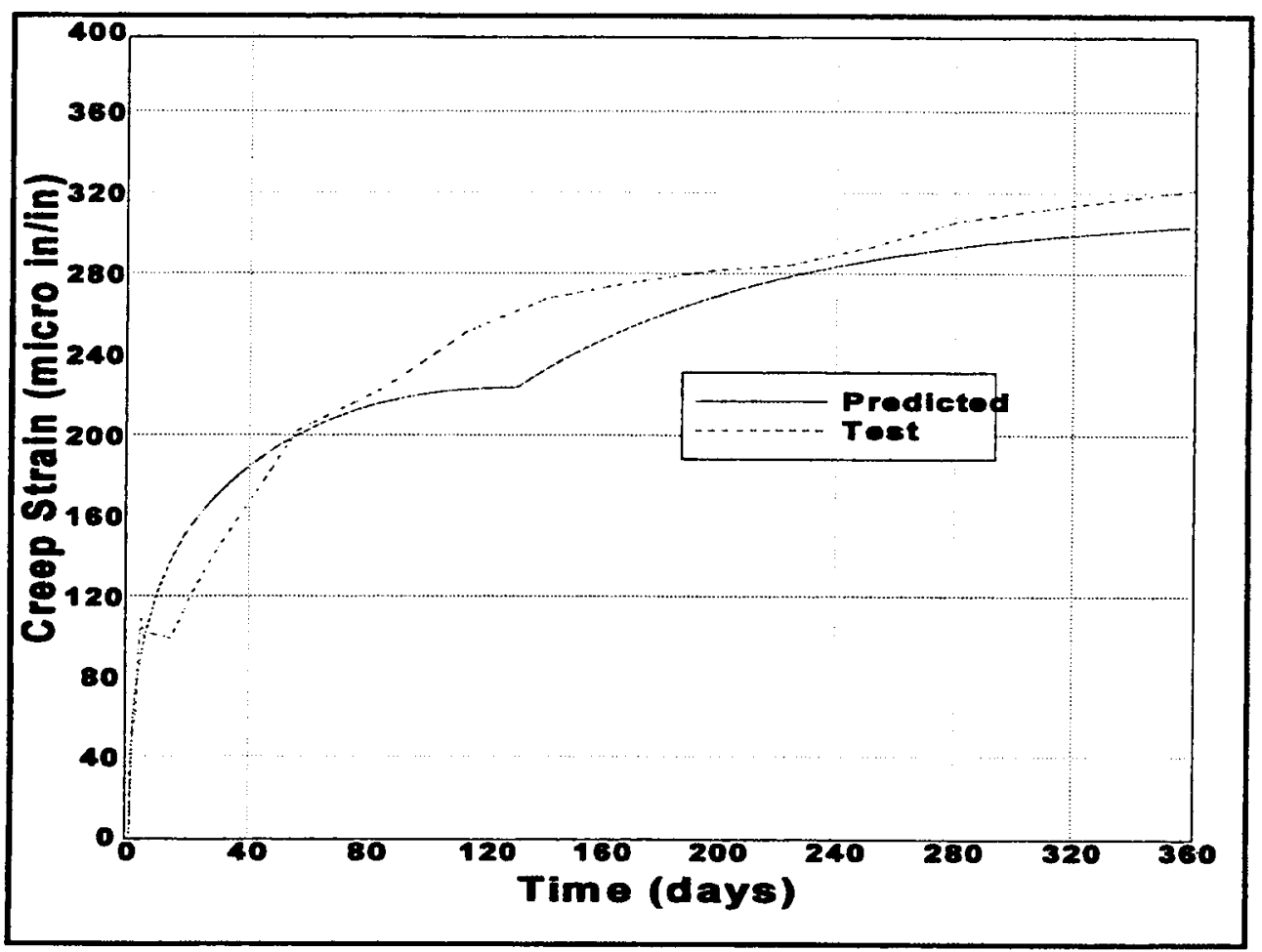

Figure 3. Test and predicted strains, B-7

\section{FE Analyses Results}

Only two biaxial tests are listed in Table 1: test F-13, with a 600-psi radial pressure and test H-22, with a 3,600-psi radial pressure. Test creep strains and creep strains predicted by the FE analysis for the 600 psi test compare well in both the radial and axial directions, and are shown in Figure 4. Predictions for the 3,600-psi test could be expected to be much different from test strains for two reasons. First, the Von Mises stress of 3,600 psi is approaching a limiting value at 90 days and could result in a shear failure if predicted compressive strength was significantly less than test strength. Secondly, the creep model in the ANACAP-U is designed to predict creep for loadings of up to $0.4 f^{\prime}{ }_{c}$, or approximately 2,500 psi at day 90 . In this range, creep is assumed to be linear, and a single creep curve can be used to define creep under any loading and translated in time to define creep at any age. At higher loadings this linear creep relationship is not applicable. To ensure that failure did not occur, the 3-day compressive strength was increased from 1,260 psi, the McAlpine value, to 4,260 psi for the analysis modeling this test. Analysis predictions for this test were much lower than test creep strains (Figure 5) due to the nonlinearity of creep at this stress level. The assumption of the linearity of creep is valid for mass concretes, where high compressive stresses are unusual, and the stress range of interest is normally limited by the tensile capacity of the concrete, but may result in errors in predicted creep strains if the ANACAP-U aging viscoelastic model used in these analyses is used to model structural concrete, which can experience high compressive stresses. 


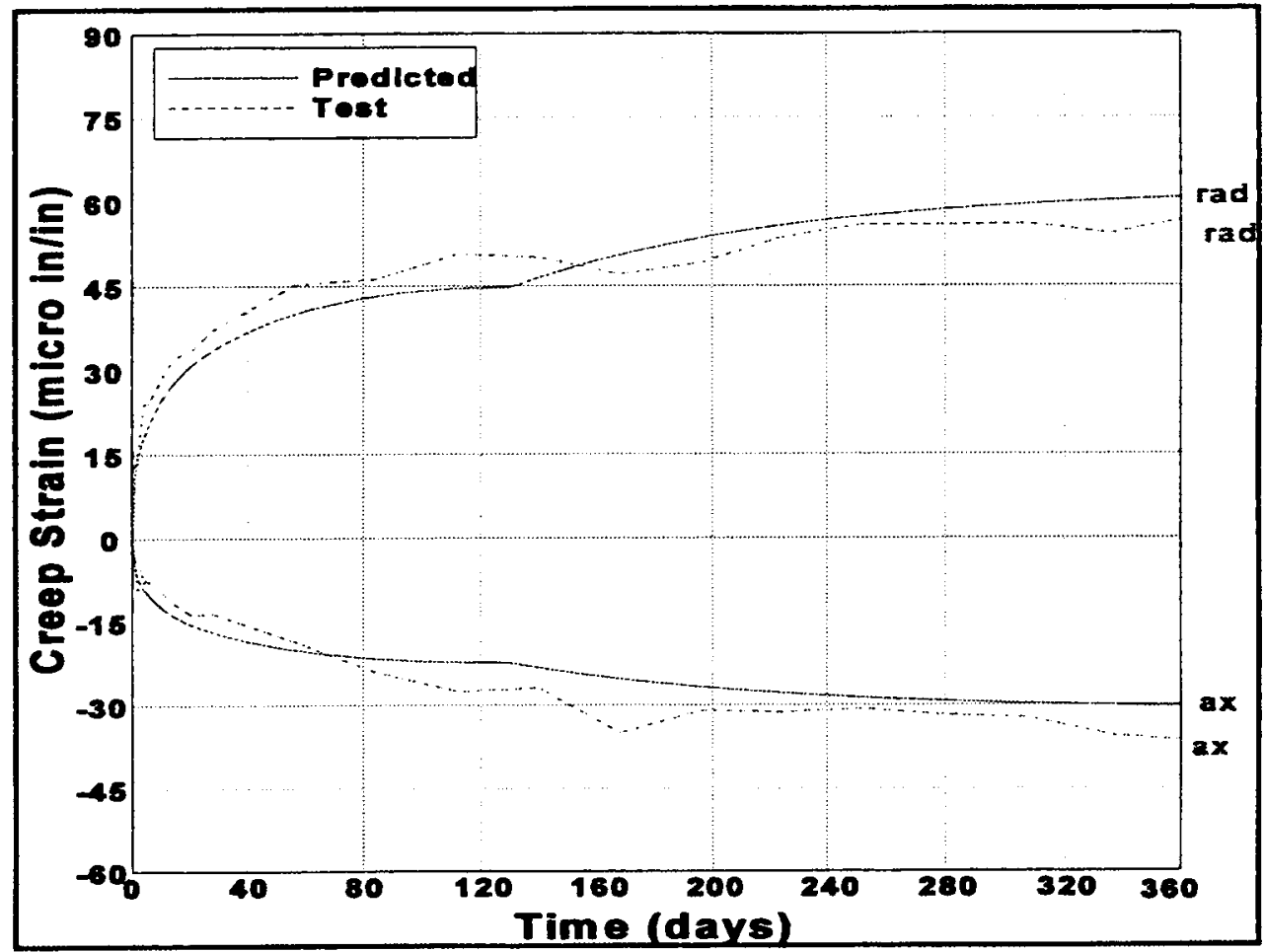

Figure 4. Test and predicted strains, 600-psi biaxial loading (F-13)

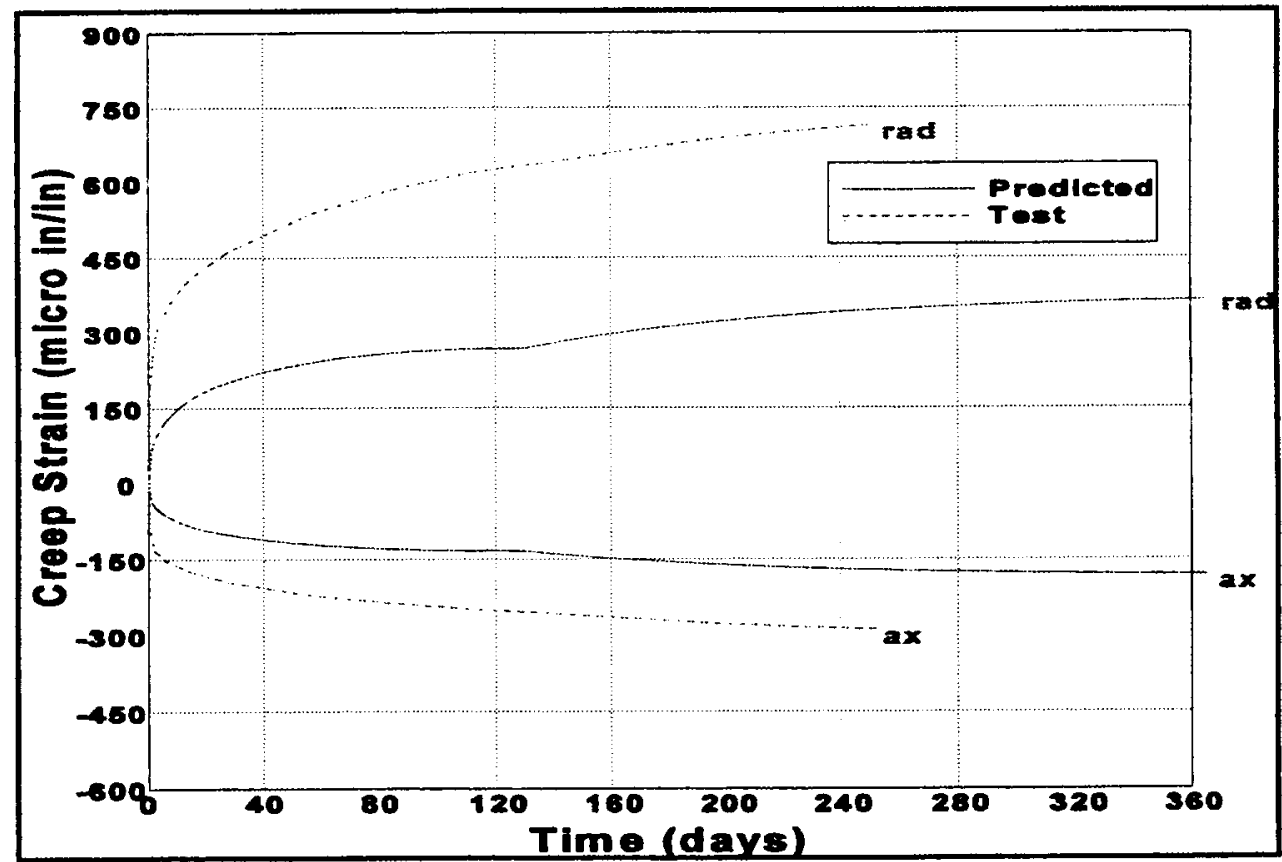

Figure 5. Test and predicted strains, 3,600-psi biaxial load 
Predicted creep strains for the triaxial test simulations agreed well with test strains in tests C-23 and D-26, in which radial stresses were low when compared with axial stresses. Predicted axial creep strain for the C-23 test on day 360 was only 13 percent greater than test axial creep strain at that time. Predicted axial and radial creep strains for test specimen D-26 were 6 and 13 percent greater than the respective test values on day 360 . Predicted and test strains for these two tests are shown in Figures 6 and 7.

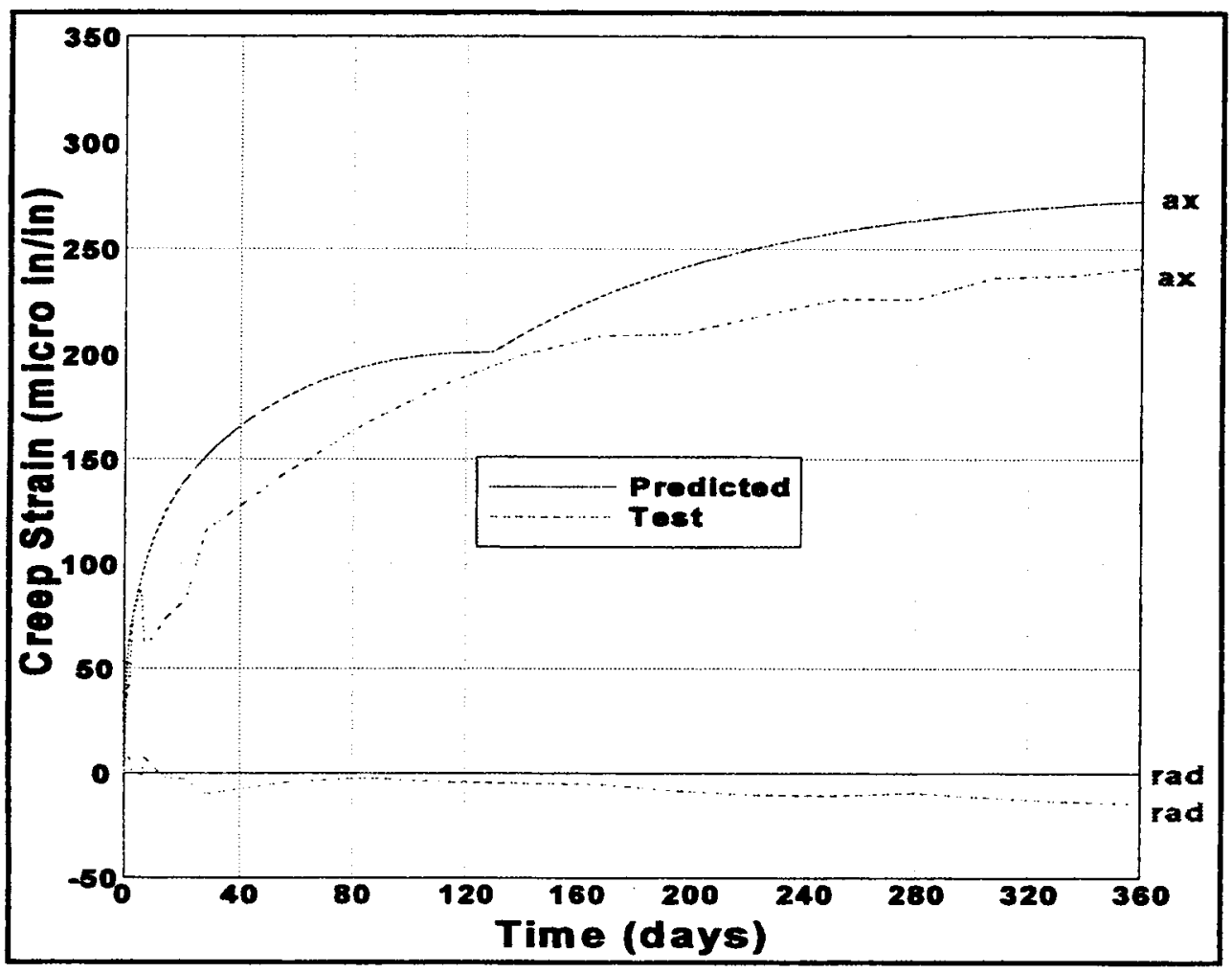

Figure 6. Test and predicted strains, 2,400-psi axial pressure, 600-psi radial pressure (C-23)

For load cases in which stresses were hydrostatic, significant differences were observed between the test and predicted strains. For all hydrostatic loadings, test radial creep strains exceeded test axial creep strains. This was in part explained by friction losses in the axial loading mechanism that resulted in actual pressures 3 to 10 percent below design values. However, radial strains exceeded axial strains by more than 10 percent in all hydrostatic tests. In test E-5, with a 600 -psi hydrostatic loading (Figure 8), predicted creep strains were 10 percent lower than test radial creep strains and 9 percent higher than text axial creep strains at 120 days after loading. This difference is partly attributable to the fact that the design loads used in the numerical test simulations were slightly different from the actual loadings due to the reduced axial loading. However, by day 360 the predicted creep strains were 23.2 percent greater than the radial test creep strain and 90.2 percent greater than the axial test creep strain, which decreased after 


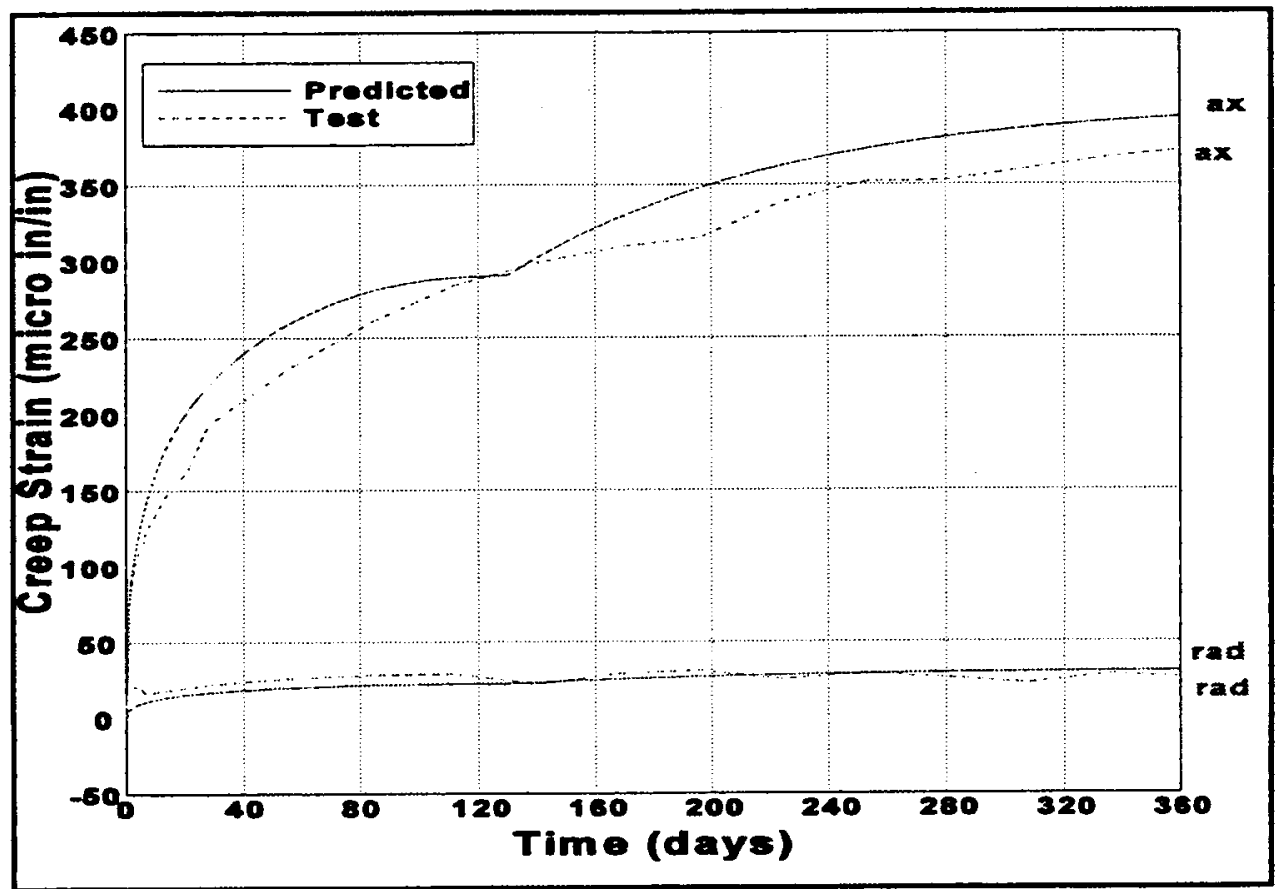

Figure 7. Test and predicted strains, 3,600-psi axial pressure, 1,200-psi radial pressure (D-26)

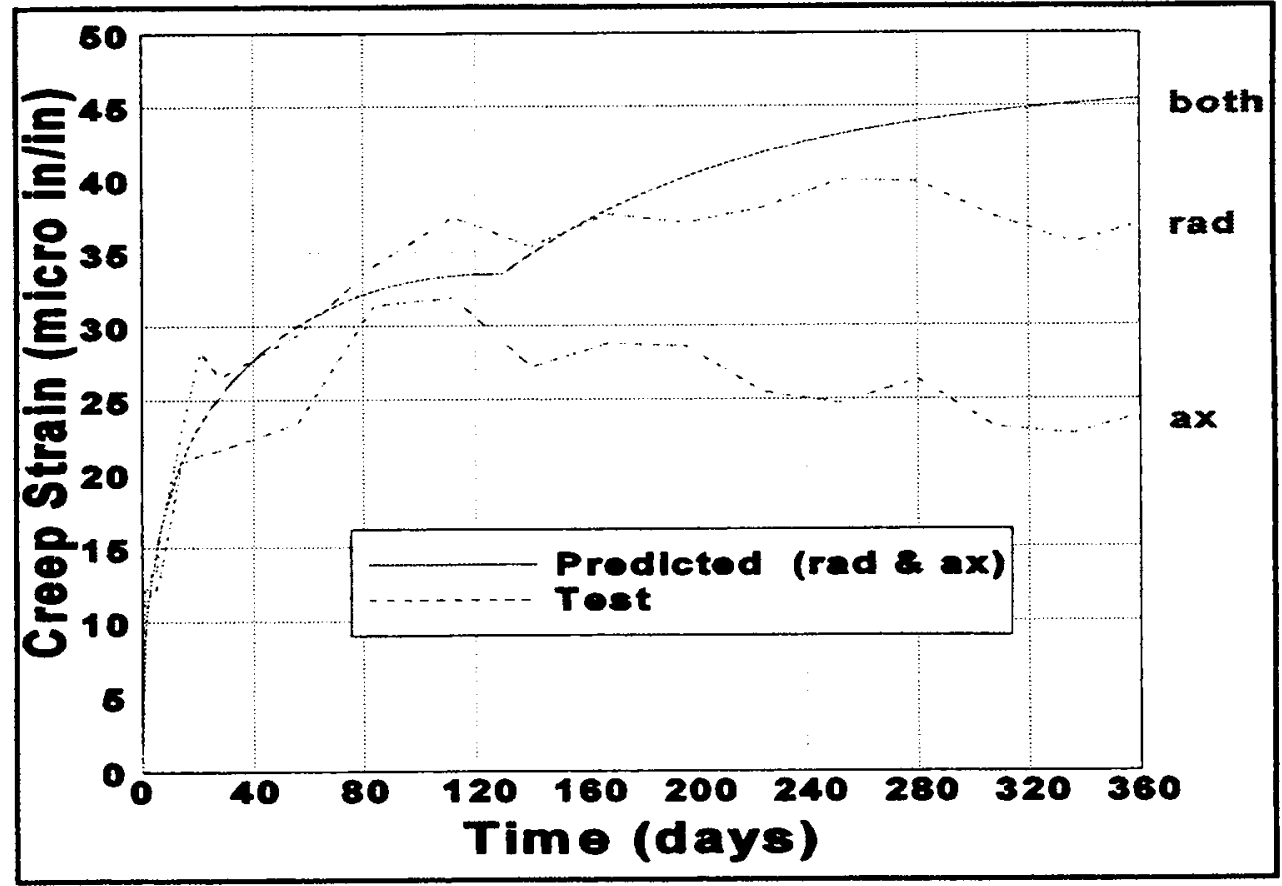

Figure 8. Test and predicted strains, 600-psi hydrostatic loading (E-5) 
day 120 . The fact that the test gauges indicated zero or decreasing creep between 120 and 360 days would seem to indicate a loss of load, but none was reported. In the 2,400-psi hydrostatic test (Figure 9), predicted axial creep strains were close to the test strains, but predicted radial creep strains were only 70 to 76 percent of the test strains. For the 3,600-psi hydrostatic test (Figure 10), predicted radial creep strains were only about 75 to 79 percent of test strains, while predicted axial creep strains were 82 to 90 percent of test strains. Predictions for this test are better than expected since the loading is outside of the range for linear creep. The level of error seen in these predictions, 10 to 30 percent, may be an acceptable level of error for creep, which is only one of the many factors that affect stresses and strains in mass concrete. In the 1,200-psi hydrostatic test (Figure 11), radial pressure was lost after $90 \mathrm{~min}$. Predicted axial creep strain for this test was 47.8 percent greater than the test strain on day 360 . In a separate analysis, a 1,200-psi uniaxial test was simulated although no uniaxial test data was available for this loading. The resulting axial creep strains were exactly equal to the strains predicted for the C-16 loading. In other words, applying the radial stress for a period of 0.0625 days $(90 \mathrm{~min}$ ) had no effect on the predicted axial strains.

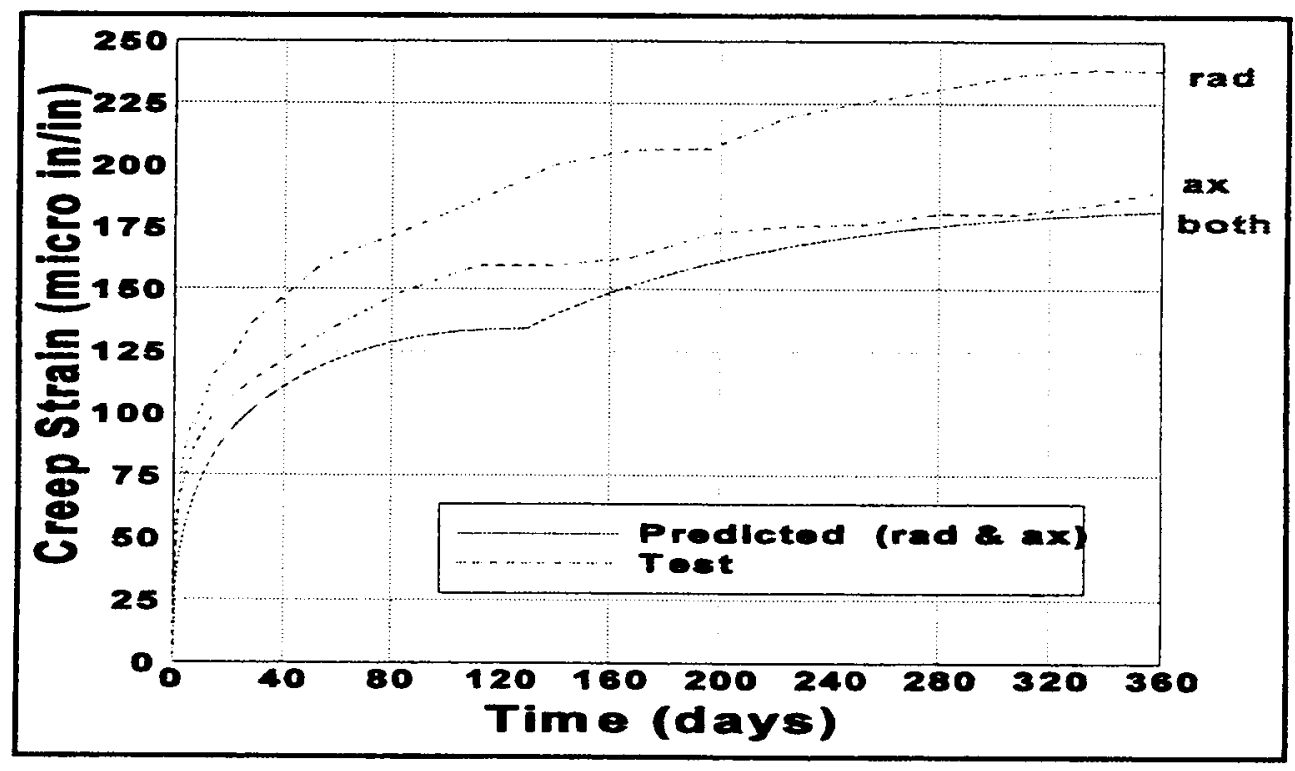

Figure 9. Test and predicted strains, 2,400-psi hydrostatic loading (F-9)

The largest differences in predicted and test strains occurred in tests B-41 (Figure 12) and G-35 (Figure 13). In test B-41, with a 2,400-psi radial pressure and 1,200-psi axial pressure, test axial creep strains were much larger than test radial creep strains. As would be expected, ANACAP-U predicted larger radial creep strains and very little axial creep strain. No explanation was provided for this apparent reversal of test creep strains, although a radial pressure drop was reported on day 21 . Reported total radial strains dropped between day 0.25 and day 1 , on day 6 , and on day 21 , possibly indicating drops in radial pressure.

Since pressure histories were not reported, accurate simulation of pressure drops in the FE analyses was not possible. Specimen G-35 was loaded with a 600-psi 


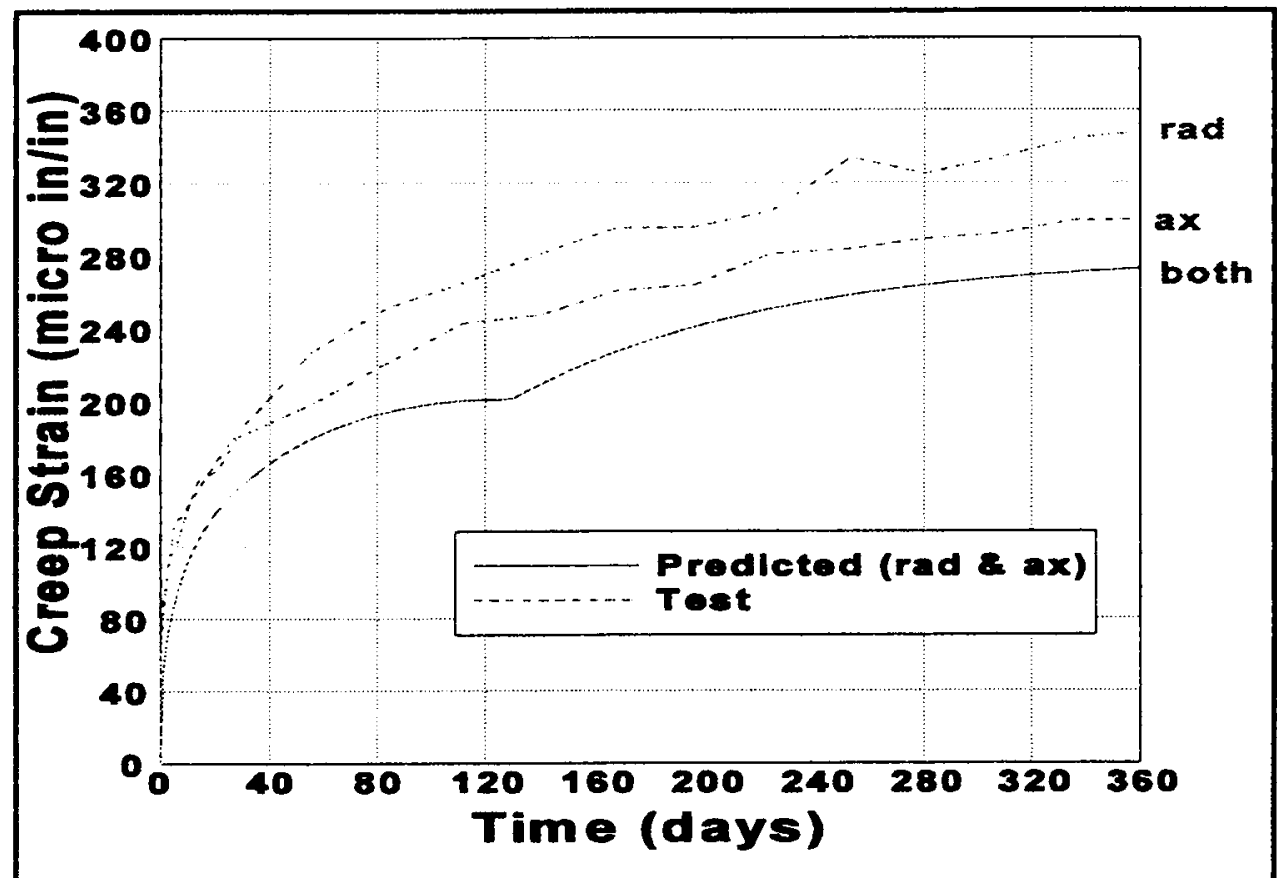

Figure 10. Test and predicted creep strains, 3,600-psi hydrostatic loading (D-31)

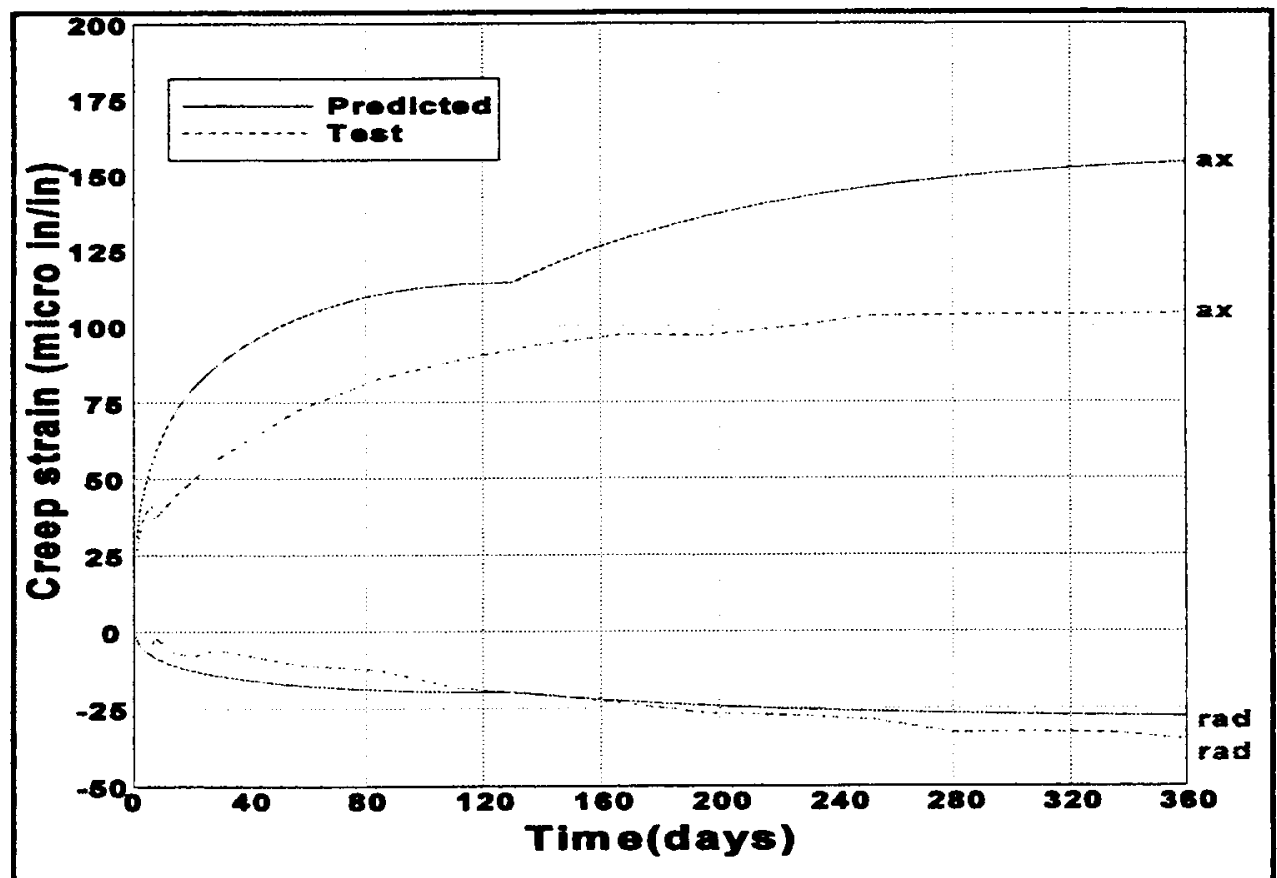

Figure 11. Test and predicted creep strains for 1,200-psi hydrostatic loading with loss of radial load after 90 min (C-16) 


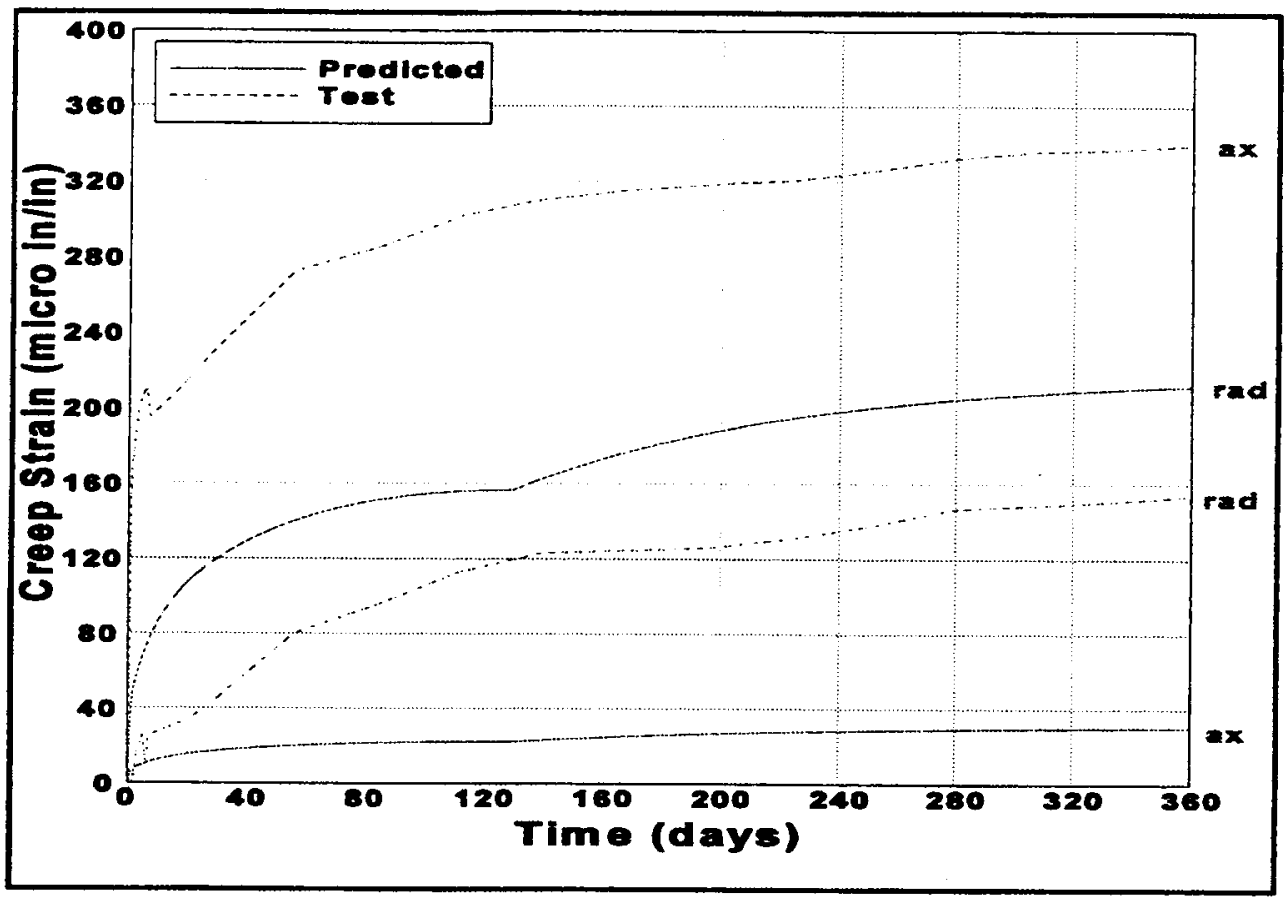

Figure 12. Test and predicted strains for 1,200-psi axial loading, 2,400-psi radial loading (B-41)

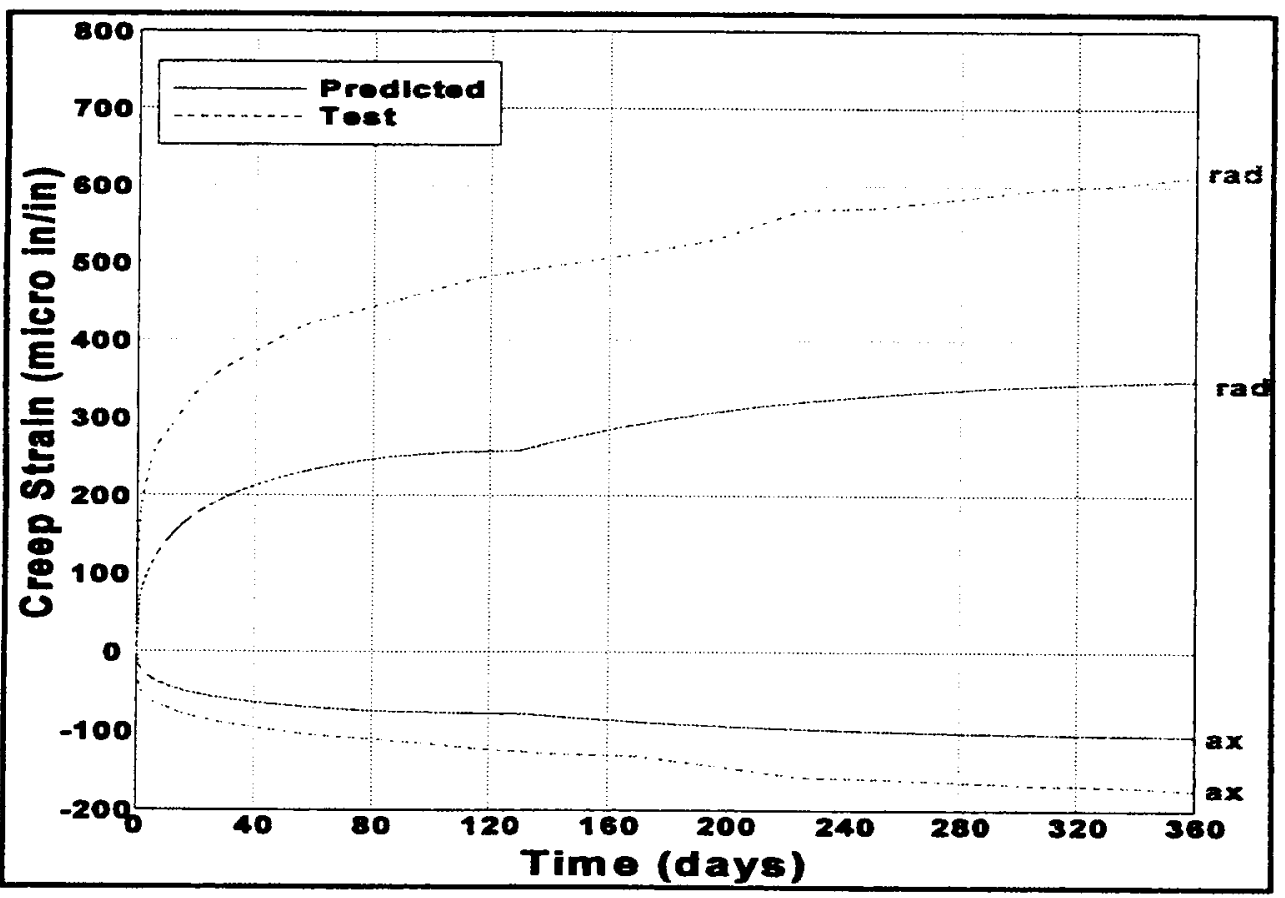

Figure 13. Test and predicted creep strains for 600-psi axial loading, 3,600-psi radial loading (G-35) 
axial stress and 3,600-psi radial stress. Although the Von Mises stress for this condition was only 3,000 psi, the analysis 3-day compressive strength was increased to 4,260 psi to ensure that shear failure did not occur. Test radial creep strains for this specimen were 75.8 percent greater than ANACAP-U radial creep strains on day 360 , and test axial strains were 65 percent greater than ANACAP-U strains. However, 3,600-psi is approximately 56 percent of the 90-day compressive strength for specimen G-35 and is outside the range of stresses for which creep can be considered to be linear.

Two tests by McDonald (1973) were also modeled. The 2,400-psi axial test was used to calibrate the material model, and a triaxial test with 2,400-psi axial pressure and 600-psi radial pressure was analyzed using the calibrated model. Predicted axial strain for the triaxial $\mathrm{FE}$ analysis was 13 percent greater than test axial strain on day 360 . Analysis radial strains were slightly higher than test radial strains, which were close to zero. Calculated and test strains for the two tests are compared in Figures 14 and 15.

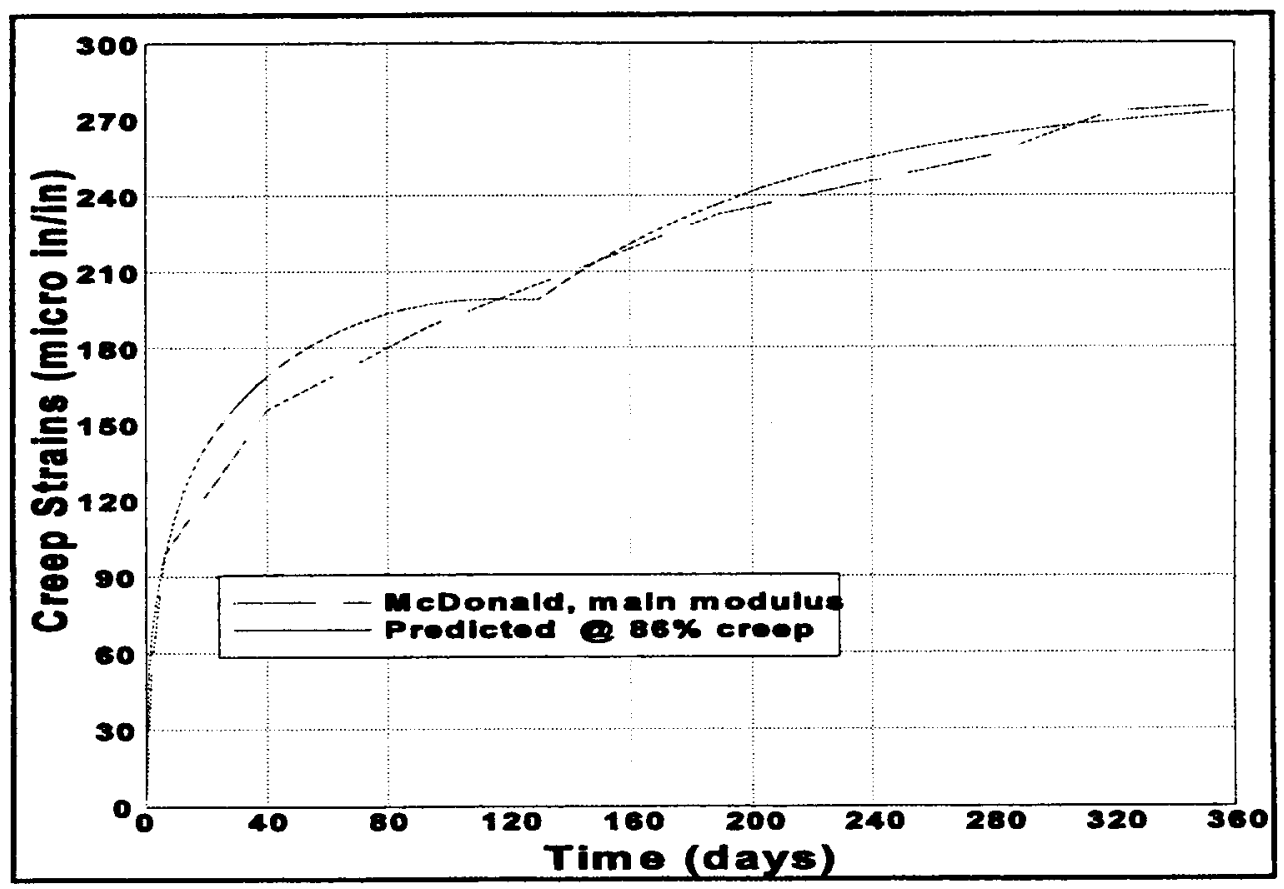

Figure 14. Test and predicted strains, 2,400-psi axial creep test, McDonald main modulus concrete

\section{Conclusions}

The creep model in the ANACAP-U viscoelastic aging concrete model is based on the linearity of creep for loadings in the range of 0 to 40 percent of the compressive strength of the concrete. This assumption is certainly applicable to the prediction of stress and strain in mass concrete structures, which normally 


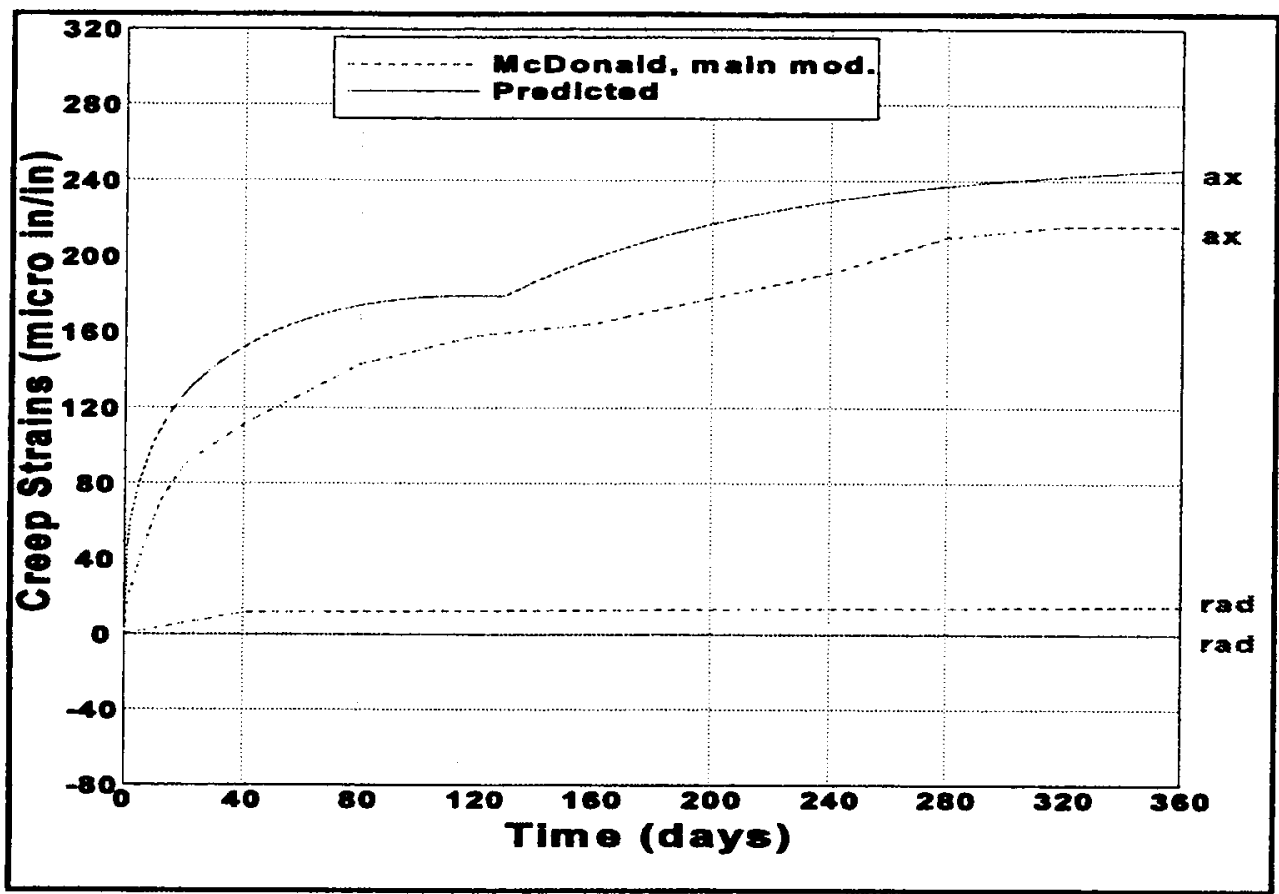

Figure 15. Test and predicted creep strains, triaxial test, McDonald main modulus concrete

experience loadings much lower than the 40 percent upper limit. Based on the multiaxial test data of McDonald (1973) and York, Kennedy, and Perry (1970), calibration of the model to uniaxial data yields acceptable predictions for biaxial and triaxial states of stress that fall within the range for which the linear creep model was intended. At loadings greater than $\mathbf{4 0}$ percent of the concrete compressive strength, creep, as expected, was highly nonlinear and strains predicted by the model were much lower than test strains. 


\section{Creep Strain Recovery}

\section{Background}

As discussed, the creep model in the ANACAP-U viscoelastic aging model relies on the linearity of creep under all states of stress. This means that the same creep curve used for loading is also used to predict change in creep strain due to unloading. However, it has been observed in WES creep tests in which the loading was lost due to leaks in the hydraulic system or power failures, that the amount of strain recovered upon unloading seemed much smaller than that which might be expected for such tests. A 7-day creep test in which the loading was lost on day 8 is shown in Figure 16. This test was not modeled in this series of verification tests due to lack of necessary test data, but the small amount of creep strain recovery can be observed in the figure. This may be due in part to the fact that the concrete was a high fly-ash mixture that was not expected to mature until approximately 90 days, so any loading occurring at early ages could result in a permanent set that could not be predicted by the ANACAP-U model.

The creep tests described in the previous chapter (York, Kennedy, and Perry 1970) were conducted on mature concrete and were monitored for 90 days after the loading was removed. These tests have been used to test the accuracy of the ANACAP-U creep strain recovery predictions. In each of the tests, the creep cylinder was loaded for 1 year, at which time the load was removed, and strains were monitored for an additional 3 months after unloading to provide information on creep strain recovery.

\section{FE Modeling of Creep and Creep Recovery}

For stresses less than 40 percent of concrete strength, concrete is commonly treated as an aging viscoelastic material. Creep in this stress range is assumed to be linear. This means that at any point in time strain is proportional to stress, and creep is completely defined by the creep compliance curve. The creep compliance curve, as defined for a mature concrete by ASTM C 512-87 (ASTM 1992a), is the strain per unit of stress resulting after all shrinkage and thermal strains and the initial elastic strain have been subtracted from the total strain of a loaded specimen. For use in the aging viscoelastic model, in which modulus and therefore elastic strain changes with time, this definition of specific creep has been 


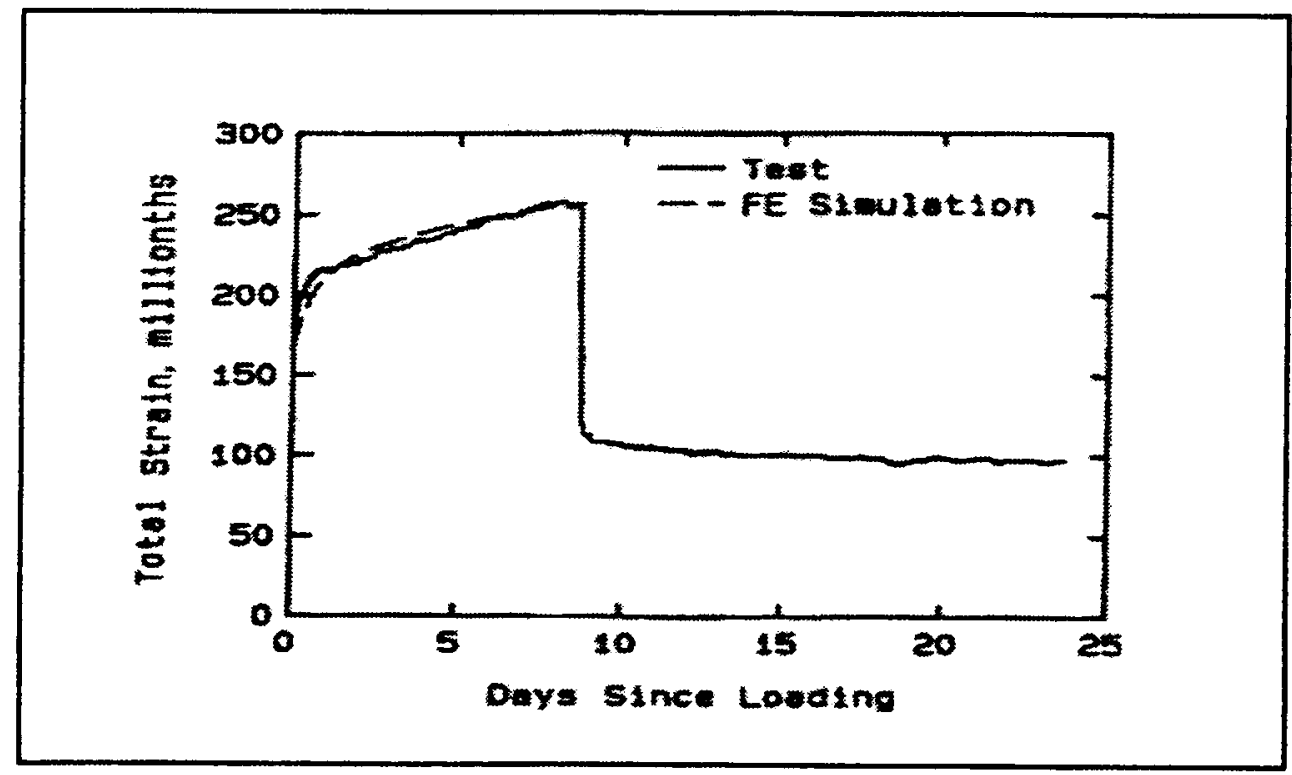

Figure 16. 7-day creep test with loading lost on day 8 (Gamer, Hammons, and Bombich 1991)

modified by subtracting the changing elastic strain due to a given loading from the total strain.

The assumption of linearity allows the application of the principle of superposition (Bazant 1988). Using this principle, current creep strain can be obtained as a hereditary integral over the stress history, and creep is recoverable to the extent that creep recovery is limited by aging. A typical creep compliance curve is shown in Figure 17 (Truman and Fehl 1995). In this figure, creep compliance for a non-aging material is given by $J\left(t-\tau_{\partial}\right)$, and creep compliance for an aging material is given by $C\left(t-\tau_{0}\right)$. A discussion of the extension of the uniaxial creep relationship to the multiaxial creep equations used in ANACAP-U may be found in the ANACAP-U theory manual (ANATECH Research Corporation 1992a).

One of the limitations of the principle of superposition discussed by Bazant (1988) is that it does not provide accurate predictions for a decrease in the strain magnitude due to unloading. However, in practice superposition is commonly used to predict strains during unloading even though this may result in some loss of accuracy. Also, if stresses are changed very slowly, the use of the principle of linear superposition is generally considered to predict creep strains due to stress fluctuations satisfactorily (Bazant 1988).

In the FE analyses, the effects of creep strain recovery were simulated by extending the analyses (discussed in the previous chapter) for 90 days after removal of the loadings. For purposes of comparison between predicted and test curves, creep strains were set equal to zero immediately after unloading in all curves. Only changes in strain after unloading were considered. 


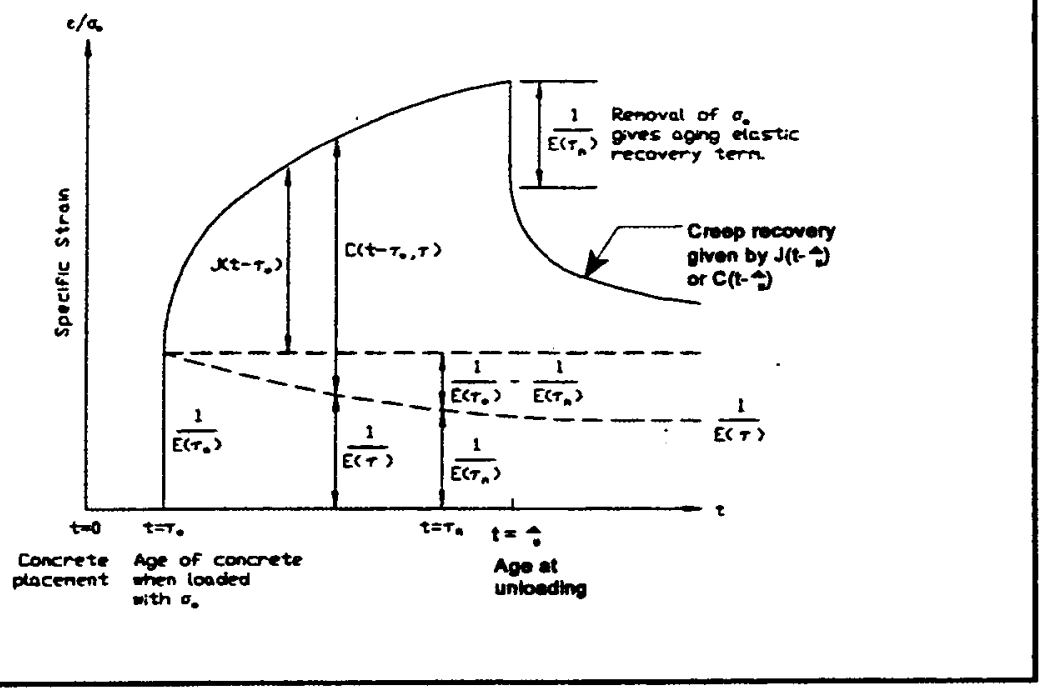

Figure 17. Typical specific creep curves, aging and non-aging materials (where $E=$ modulus of elasticity)

\section{Analyses Results}

Comparisons between predicted strains after unloading and test curves for the uniaxial and biaxial tests are shown in Figure 18, and those for the hydrostatic tests are shown in Figure 19. In the uniaxial and biaxial plots, predicted creep recovery strains in the direction of loading were between 200 and 300 percent of test strains. For the hydrostatic tests, predicted creep strain recovery was close to three times the test creep strain recovery in each of the three tests modeled.

Predicted and test creep recovery strains for three of the triaxial tests are shown in Figure 20. In tests C-23 and D-26, radial pressures (600 and 1,200 psi respectively), were small when compared with axial pressures $(2,400$ and 3,600 psi, respectively) and both test and predicted strain recoveries were small. Predicted axial creep recovery for these cylinders was greater than 200 percent of the test values. In test G-35, axial pressure (600 psi) was small when compared with radial pressure ( $3,600 \mathrm{psi})$, and predicted radial creep recovery was greater than 200 percent of the test values.

In Figure 21, predicted and test axial creep recovery strains for test E-39, the 600-psi uniaxial test, are plotted on a positive scale with test and predicted creep strains under loading for purposes of comparison. Predicted and test creep strains at 80 days after loading and predicted and test creep recovery strains at 80 days after unloading are given in Table 2. Predicted axial creep recovery strains were similar to predicted axial strains under loading, as would be expected for a mature concrete and a linear creep model, while test axial creep recovery strains were only about one-third of the strains predicted under loading. 


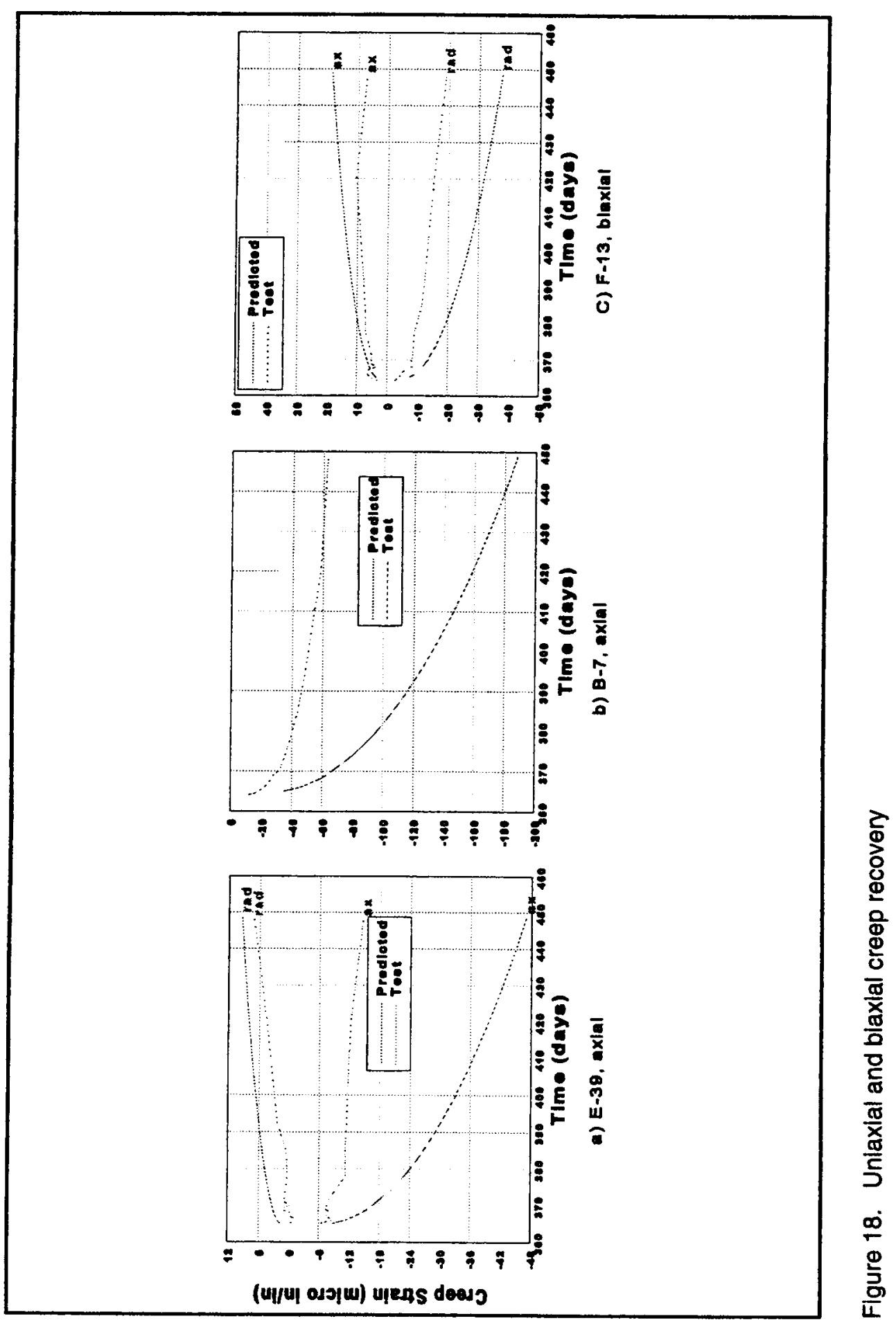




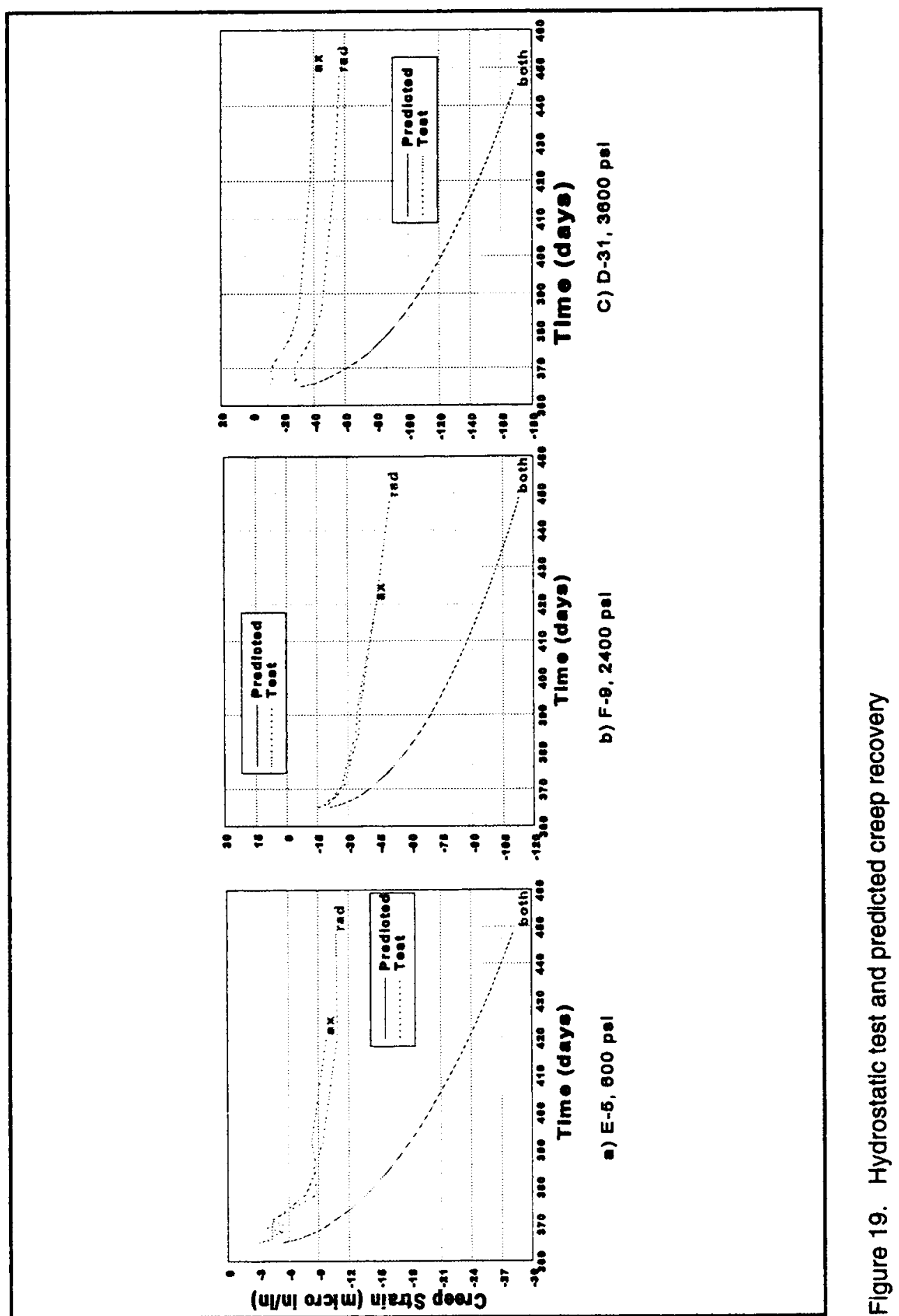




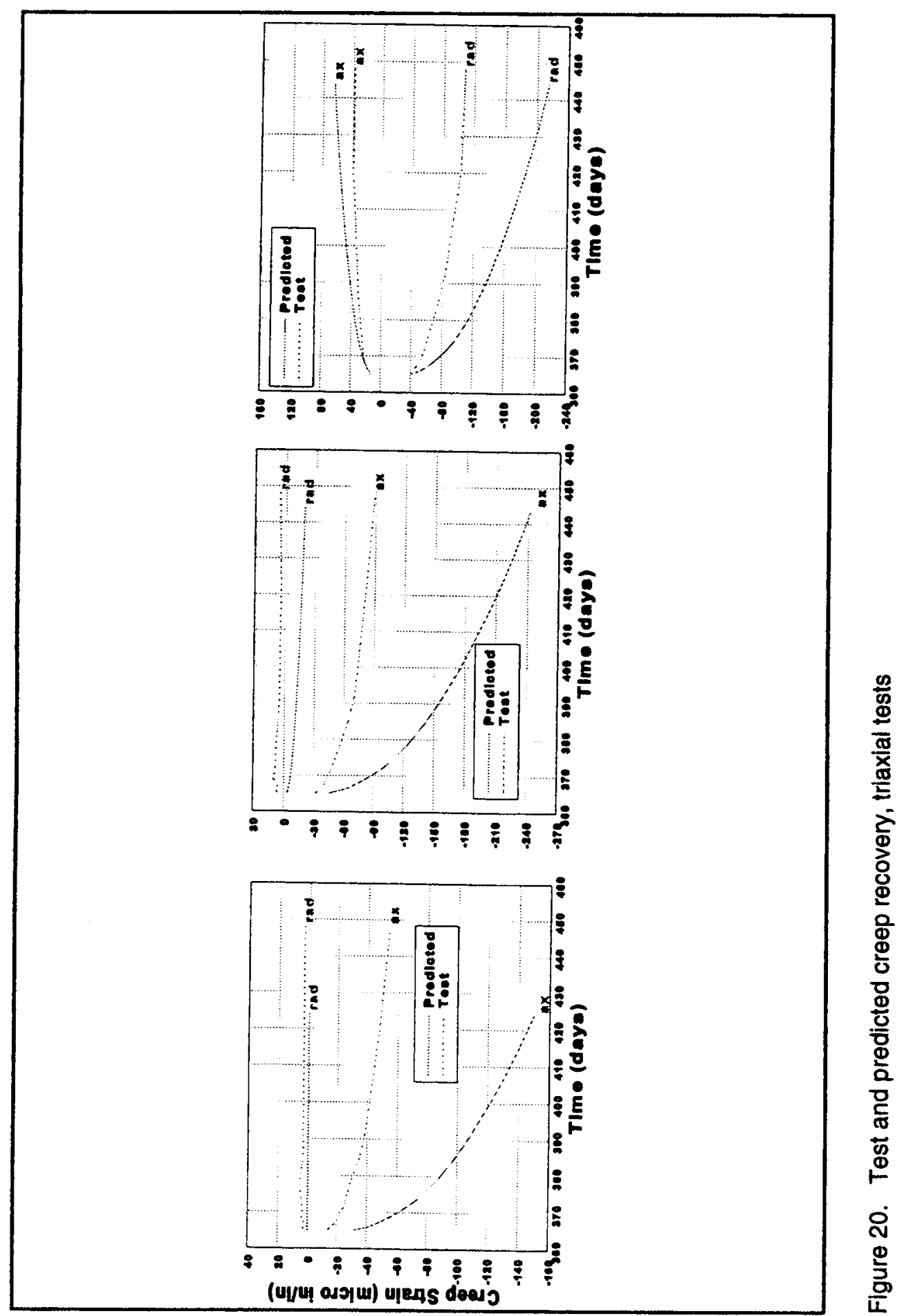




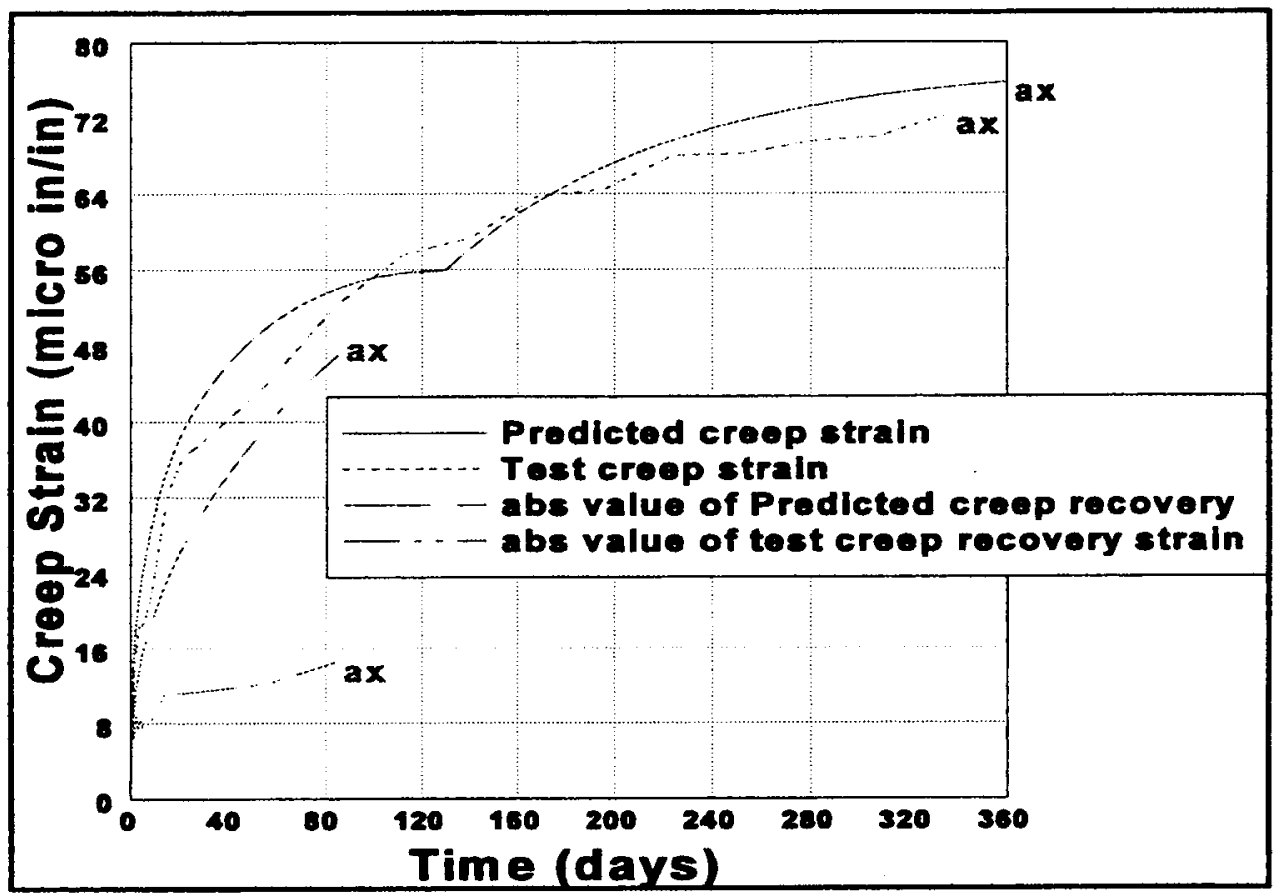

Figure 21. Test and predicted creep and creep recovery strains, E-39

This plot demonstrates the shortcomings of the linear creep model in cases of dramatic unloading.

\begin{tabular}{|c|c|c|c|c|c|}
\hline \multicolumn{6}{|c|}{$\begin{array}{l}\text { Table } 2 \\
\text { Creep and Creep Recovery, Test E-39 }\end{array}$} \\
\hline \multicolumn{4}{|c|}{ Absolute Value of Creep Strains, micro in $/ \mathrm{in}$. } & \multicolumn{2}{|c|}{$\begin{array}{l}\text { Creep Recovery as } \\
\text { Percentage of Creep }\end{array}$} \\
\hline \multicolumn{2}{|c|}{80 Days After Loading } & \multicolumn{2}{|c|}{80 Days After Unloading } & \multirow[b]{2}{*}{ Predicted } & \multirow[b]{2}{*}{ Test } \\
\hline Predicted & Test & Predicted & Test & & \\
\hline 53.58 & 51.095 & 45.92 & 14.171 & 85.7 & 27.7 \\
\hline
\end{tabular}

\section{Conclusions}

The large differences between test and predicted creep recovery demonstrated in the figures above occur under circumstances for which the principle of superposition could not be expected to provide accurate predictions. However, these conditions do not typically occur in a thermal stress analysis of young concrete. Strains in mass concrete are normally induced by relatively slowly changing loadings. Thermal loadings are usually provided by the average ambient temperature curve and by the adiabatic temperature rise of the material. For a thick wall placed during the warmest part of the year, temperatures near the center rise significantly above ambient air temperatures due to the heat generated by the hydration of the cementious materials, while surface temperatures remain much 
closer to the ambient air temperature. This process creates a temperature gradient between the outer surface and the center of the wall, resulting in expansion at the center and contraction at the surface as ambient temperatures fall. These surface contractions are restrained by the large mass of heated interior concrete and by the thermal gradient, resulting in tensile stresses at the surface. These surface tensile stresses are somewhat relieved by the reciprocal effect of creep, stress relaxation. Under the conditions described, maximum tensile stresses should occur near the minimum of the ambient temperature curve. Surface stresses will then slowly decrease as the average ambient temperature rises. The ANACAP-U model should be able to represent creep and stress relaxation fairly accurately under the conditions described. However, creep predictions may become less accurate for a sudden drop in loading since creep recovery will be overpredicted.

At present there is no consistent data to justify using anything other than a viscoelastic creep model in cases of unloading. At least one series of creep tests in which the loading is gradually reduced with time and one series in which the loading is reduced in a series of sudden steps would provide data for determining the level of accuracy of the current model under these conditions, and possibly provide data to initiate the consideration of modifications to the method of predicting creep recovery. 


\section{Slow Load Beam Test}

\section{Introduction}

The slow load beam test is used to determine the ultimate failure strain of the concrete in a pure bending mode and under slow load conditions. The ultimate failure strain and the load which created this strain are then used to establish parameters that define the threshold in the constitutive model that will be used for determining if cracking will occur in the concrete model. Since the ultimate strain capacity is the key component in crack prediction, it is important to accurately determine this parameter.

The slow load beam test is comprised of a simply supported beam equally loaded at the third points. Figure 22 shows how the test is set up as well as how the dimensions are used. The beam is loaded through the use of a hydraulic ram operated under electronic control. The loading of the beam is increased on a weekly basis until the beam fractures.

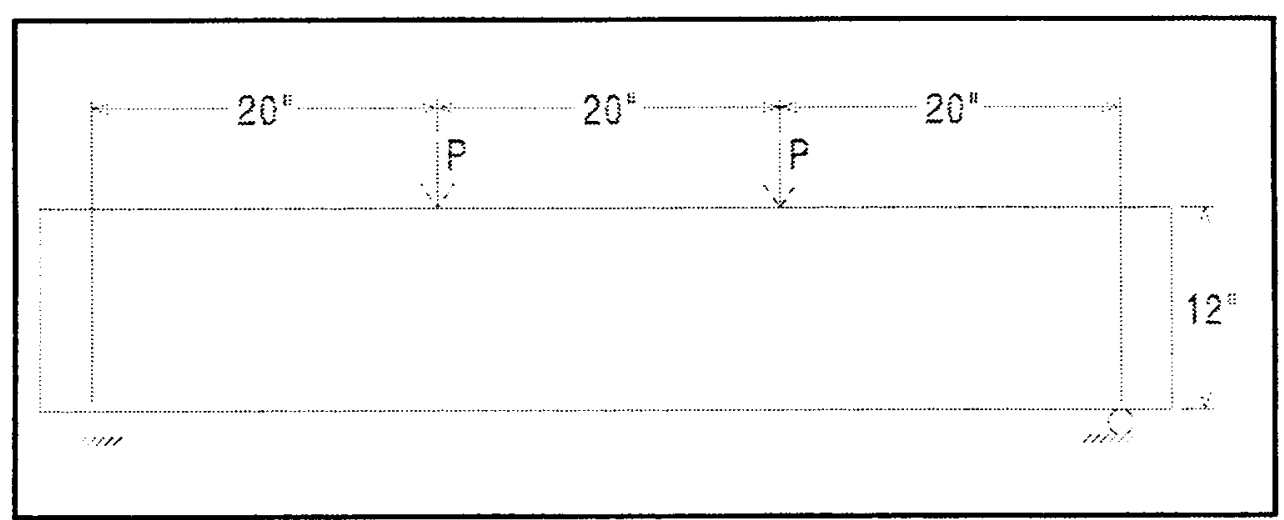

Figure 22. Geometry and loading of beam used in slow load test

Based on the time the fracture occurred and the load which created the fracture, the ultimate stress can be computed and the ultimate strain capacity can be measured. The threshold of cracking used in the constitutive model is an interaction of strain and stress as shown in Figure 23. The ultimate fracture strain $\epsilon_{f}$ is the anchor point on the horizontal axis of the interaction diagram, while the ultimate fracture stress $\sigma_{f}$ varies with time as a function of the modulus of 


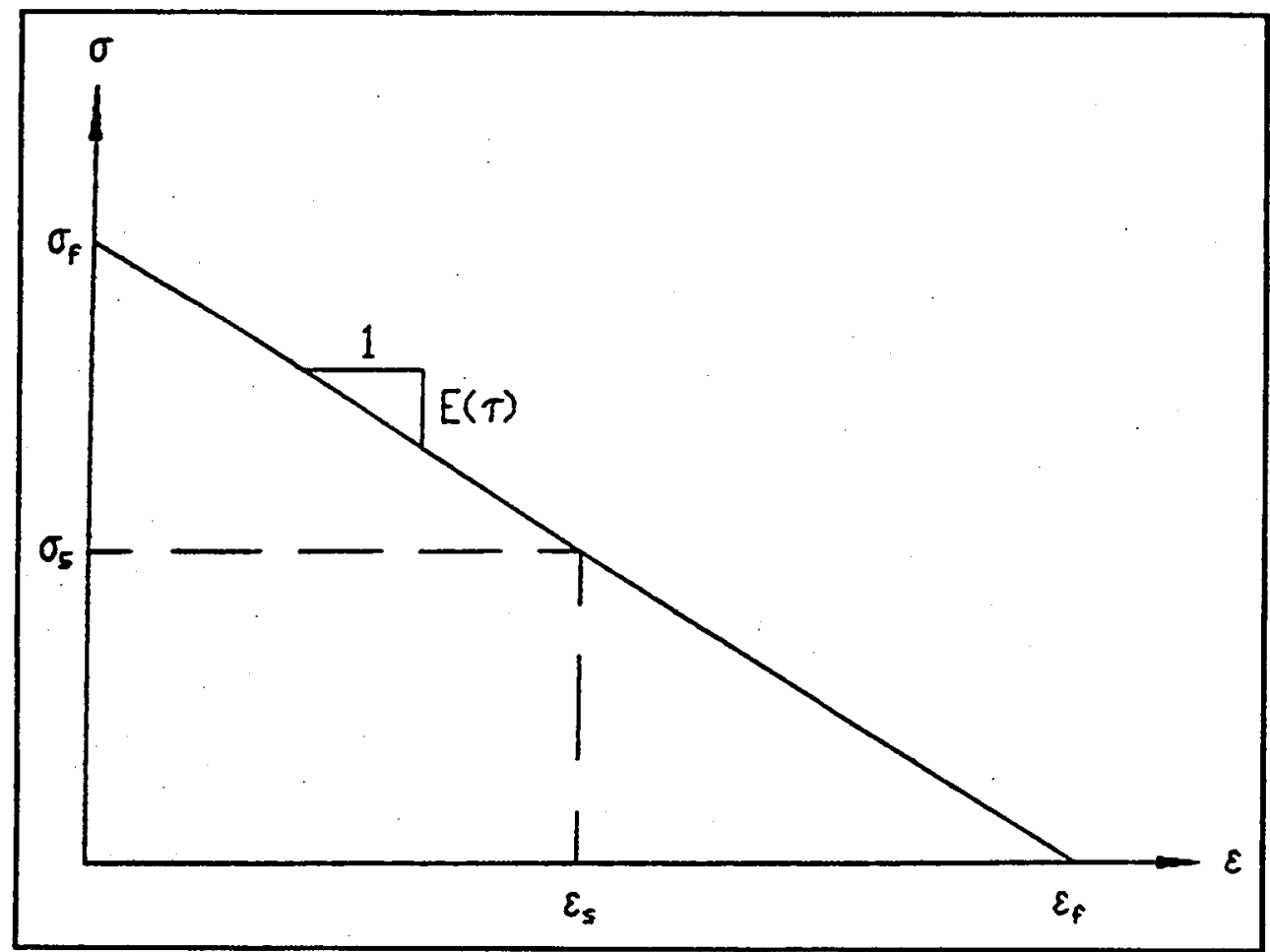

Figure 23. Stress-strain interaction diagram used as cracking threshold in constitutive model $\left(\sigma_{\mathrm{s}}=\right.$ stress at the bottom of the beam; $E(\tau)=$ modulus of elasticity at time $\tau$ )

elasticity. In the figure $\epsilon_{f}$ is a fixed point while $\sigma_{f}$ will vary with time since it is related to the modulus of elasticity $E$ through the following equation:

$$
\sigma_{f i}=\epsilon_{f} E\left(t_{i}\right)
$$

where $t_{i}$ is the time elapsed since the time of set. Therefore, the threshold as presented in Figure 23 is for one instant in time and will be different for various times. For example $\sigma_{f}$ will be less for times earlier in the life of the concrete and will be higher for times later in the life of the concrete.

The ultimate fracture strain $\epsilon_{f}$ cannot be obtained directly from the test results since it is the strain at which fracture will occur when there is no stress present. This condition will not likely occur in actual structures, but use of this point to anchor the curve to the horizontal axis shown in Figure 23 is necessary to identify the curve used to predict failure for actual conditions. Such a case is the slow load beam test and therefore $\epsilon_{f}$ must be extrapolated from the results obtained during the test. When the fracture occurs, the known parameters are the magnitude of the applied load, the age of the concrete, and the strain near the bottom of the beam (taken from strain gauges mounted on the beam). Since the center of the beam is in pure bending, the stress in the bottom of the beam (modulus of rupture $\sigma_{r}$ ) can be computed using the load at the time of fracture with the following equation: 


$$
\sigma_{r}=\frac{P_{f} a c}{2 I}
$$

where

$$
\begin{aligned}
& P_{f}=\text { total load at fracture } \\
& a=\text { distance from a support to the nearest point of applied load } \\
& c=\text { distance from the bottom of the beam to the centroid } \\
& I=\text { moment of inertia of the beam }
\end{aligned}
$$

Since the time of fracture is known, the modulus of elasticity at the time of fracture can be ascertained from the test data obtained during material testing.

Prior to computing the ultimate fracture strain of the concrete, the slow load fracture strain (strain at the bottom of the beam $\epsilon_{s}$ ), and the slow load fracture stress (stress at the bottom of the beam $\sigma_{s}$ ) must be determined. These parameters are not the value of stress and strain at actual failure but at 90 percent of failure and are used because the stress-strain relationship becomes nonlinear near failure due to microcracking occurring in the concrete. Therefore, the slow load fracture stress is $0.9\left(\sigma_{r}\right)$. A linear regression analysis is performed which relates the extreme fiber strain to the applied stress, and it is from this resulting linear regression that the slow load fracture strain can be determined based on the value of $\sigma_{s}$.

Once $\sigma_{s}, E$, and $\epsilon$, have been determined as described above, the ultimate fracture strain can be computed by using the following equation:

$$
\epsilon_{f}=\epsilon_{s}+\frac{\sigma_{s}}{E\left(t_{s}\right)}
$$

where $t_{s}$ is the time at which slow load fracture stress was achieved in the test.

Based on established parameters within the constitutive model and the ABAQUS model parameters, the stress-strain state at each integration point in the model is checked against the cracking criterion (Figure 24) to determine if any cracking is predicted. If the stress-strain state of an integration point exceeds the cracking criterion, then the stiffness at that point is reduced to zero in the direction of the maximum principal strain.

The stress-strain interaction diagram is also used to provide information about integration points which have not cracked. The constitutive model provides a cracking potential of uncracked points based on the location of the stress-strain state on the diagram relative to the stress-strain threshold. Output for cracking potential is provided in the form of a percentage. This percentage is computed by 
dividing the distance between the stress-strain state and the origin $\left(1_{1}\right)$ by the distance from the stress-strain threshold to the origin $\left(l_{1}+l_{2}\right)$ (Figure 24). The cracking potential is valuable information since it will indicate whether or not a structure is approaching cracking and can be plotted in contours to indicate areas where there may be a high potential for cracking even though the model did not actually predict cracking.

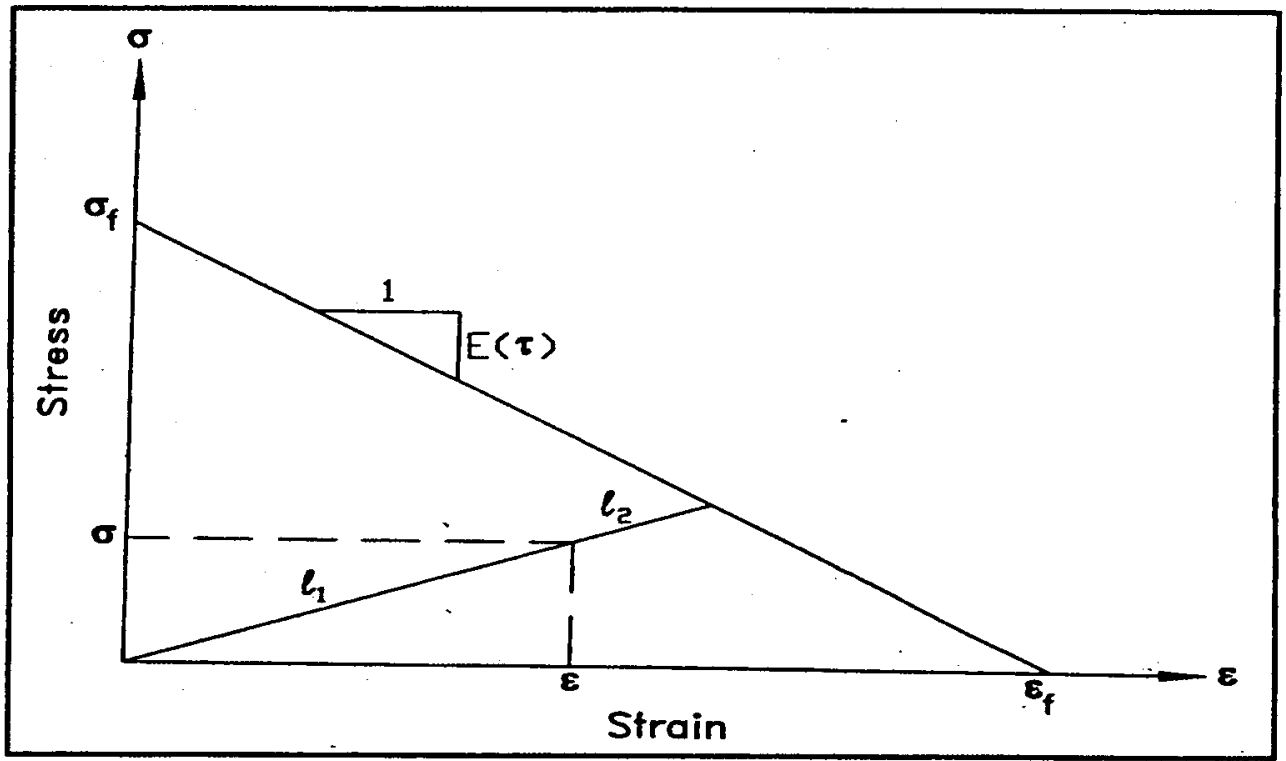

Figure 24. Cracking potential generation for a specific cracking failure surface

\section{Test}

The test setup shown in Figure 22 is based on ASTM C 78-75 (ASTM 1992b). The test specimen is 12 in. wide. The loading of the beam was based on applicable provisions of C 71-80 (USACE 1949) which specify a loading rate that will increase the stress in the extreme fiber of the beam by 25 psi per week. Based on the dimensions of the beam used for the testing of the interior mixture for the McAlpine Lock Replacement Project, this loading rate requires that a load of $720 \mathrm{lb}$ be applied weekly to the beam.

Due to various factors, the increase in the loading did not take place in exactly seven day increments as specified in ASTM C 78-75 (ASTM 1992b). Figure 25 is a plot of the load versus time showing how the prescribed loading varies when compared with the actual loading. The actual loading will be used for comparisons in this study.

The beam failed at a total load $P_{f}$ of $10,080 \mathrm{lb}$ with concrete 109 days old. Based on the known load and the fact that in the center of the beam the stress distribution is linear, the stress in the bottom of the beam just prior to failure can be computed using Equation 2. The distance $a$ is 20 in., the distance to the 


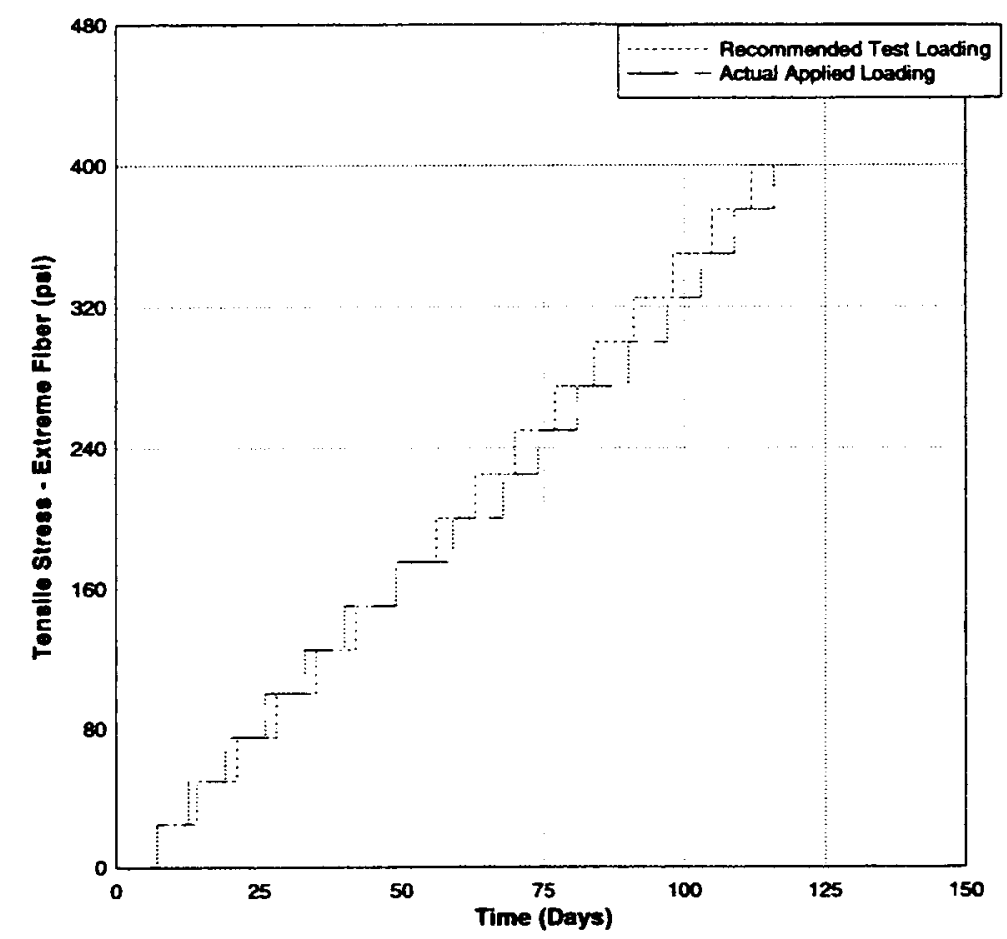

Figure 25. Comparison of recommended loading of slow load beam and actual applied loading

centroid $c$ is 6 in., and the moment of inertia $I$ is 1728 in $^{4}$. Substituting these values results in the following:

$$
\sigma_{r}=\frac{(10,080 \mathrm{lb})(20 \mathrm{in.})(6 \mathrm{in} .)}{2\left(1728 \mathrm{in} .^{4}\right)}=350 \mathrm{psi}
$$

Since the modulus of rupture is $350 \mathrm{psi}$, the slow load fracture stress will be 90 percent of this value, or 315 psi. Based on a 315 -psi slow load fracture stress, the slow load fracture strain at the bottom of the beam $\epsilon_{s}$ can be obtained from the linear regression of stress and strain as $159.0 \mu \mathrm{m}$ and is reported in Bombich, Neeley, and Garner.' The modulus of elasticity of the concrete can be computed based on the equation used in the constitutive model. Under the established loading, the age of the concrete for a stress of 315 psi would be 97 days and results in a modulus of elasticity at day 97 of $4.18 \times 10^{6} \mathrm{psi}$. Based on the above values, $\epsilon_{f}$ can be computed using Equation 3 as follows:

$$
\epsilon_{f}=159.0 \times 10^{-6} \mathrm{in} / \mathrm{in} .+\frac{315 \mathrm{psi}}{4.18 \times 10^{6} \mathrm{psi}}=234.4 \times 10^{-6} \mathrm{in} / \mathrm{in} .
$$

' Op. cit. 
Since the input for ABAQUS is for the uniaxial case, the strain value needed is half of that computed or $117.2 \times 10^{-6} \mathrm{in}$./in. and represents the fracture that would be obtained from a direct tensile test.

\section{Numerical Analyses}

Numerical analyses were performed on three different meshes. Coarse, medium, and fine meshes were used and are shown in Figure 26. The coarse mesh used elements that were 4 in. by 4 in., the medium mesh used elements that were 3 in. by 3 in., and the fine mesh used elements that were 2 in. by 2 in. All three meshes modeled only the $60 \mathrm{in}$. of beam located between the supports, so each mesh is 60 in. by 12 in. The three different meshes were used in an attempt to determine the varying effect of mesh size on the results.

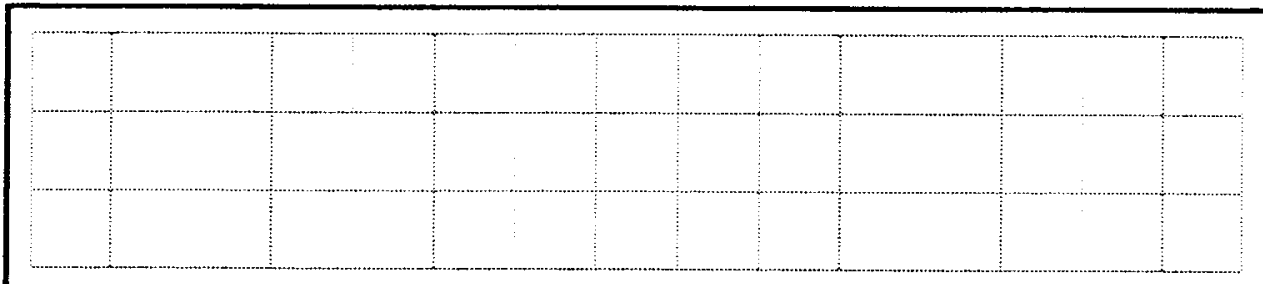

a. Coarse mesh.

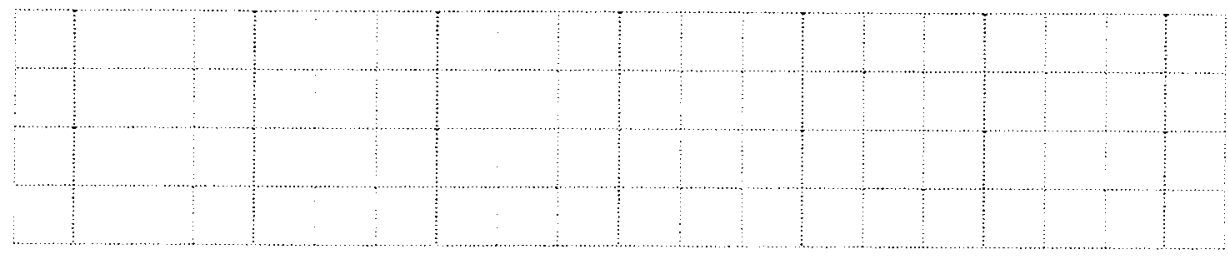

b. Medium mesh.

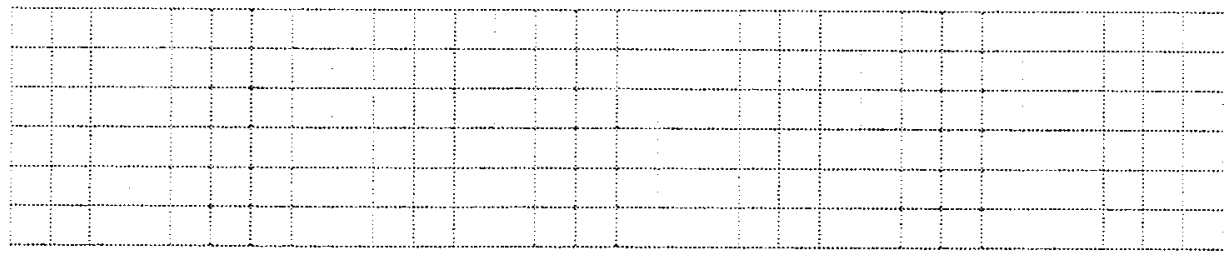

c. Fine mesh.

Figure 26. Meshes used to analyze the slow load beam test

Initially, analyses were performed on each of the three meshes using half of the ultimate fracture strain $\epsilon_{f}$ of $117.2 \mathrm{in}$./in. This resulted in a predicted failure at three different times of the analysis as shown in Table 3. As should be expected, the coarse mesh predicted the latest day of failure since all three meshes assumed the same ultimate fracture strain. Two factors affect this 


\begin{tabular}{|c|c|c|c|}
\hline \multicolumn{4}{|c|}{$\begin{array}{l}\text { Table } 3 \\
\text { Predicted Time of Failure of Slow Load Beam Test Based on } \\
1 / 2 \epsilon_{f}=117.2 \text { in. } \mathrm{in} .\end{array}$} \\
\hline Mesh Density & Coarse & Medium & Fine \\
\hline Predicted day of failure & 90.5 & 86.0 & 81.5 \\
\hline
\end{tabular}

behavior. The distribution of strain is linear from the top to the bottom of the beam, and integration points are farther away from the extreme tensile fiber for coarse meshes when compared with fine meshes. Therefore, it will require more load for the coarse mesh to attain the level of strain that occurs in the medium and fine meshes and likewise when comparing the medium mesh to the fine mesh. Regardless of the mesh chosen, the resulting predicted failure occurs earlier than the actual failure in the laboratory in all three cases, which indicates a conservative application of the failure criteria.

In order to assess the failures predicted by each of the three meshes, the analyses were performed again for each mesh except that the value used for half of the ultimate fracture strain was adjusted. This adjustment compensated for the location of the integration point with respect to the bottom of the beam so that when the strain at the extreme fiber reached the actual one-half of $\epsilon_{f}$ value, the strain at the integration point location would correspond to the fracture strain. The adjusted values for one half of $\epsilon_{f}$ were computed based on the assumption of linear strain distribution and by using similar triangles. Table 4 shows the adjusted values of one half $\epsilon_{f}$ used in the analyses and the predicted day of failure for each mesh. As might be expected, all three meshes predict failure of the beam on the same day. As in the previous case though, the failure occurs at much earlier times in the analyses than in the laboratory.

\section{Table 4}

Predicted Time of Failure of Slow Load Beam Test Based on Adjusted Values of $1 / 2 \epsilon_{f}$ in.

\begin{tabular}{|l|l|l|l|}
\hline Mesh Density & Coarse & Medium & Fine \\
\hline Adjusted 1/26, (in./in.) & 100.7 & 104.8 & 108.9 \\
\hline Predicted day of failure & 74.5 & 74.5 & 74.5 \\
\hline
\end{tabular}

Time-histories of strain measurements taken from two locations on the beam during the test are plotted in Figures 27 and 28. Also plotted in Figure 27 are the predicted total strain histories from the numerical analyses which used a constant value of $117.2 \mathrm{in} / \mathrm{in}$. for $1 / 2 \epsilon_{f}$ for all three meshes, while Figure 28 includes predicted strain histories for the analyses which used the adjusted values of $1 / 2 \epsilon_{f}$ as shown in Table 4. As can be seen, the predicted strains from the analyses correlate very well with the actual strains measured. The strain predicted by the fine mesh matches closely the strain that was measured at the bottom of the beam, 


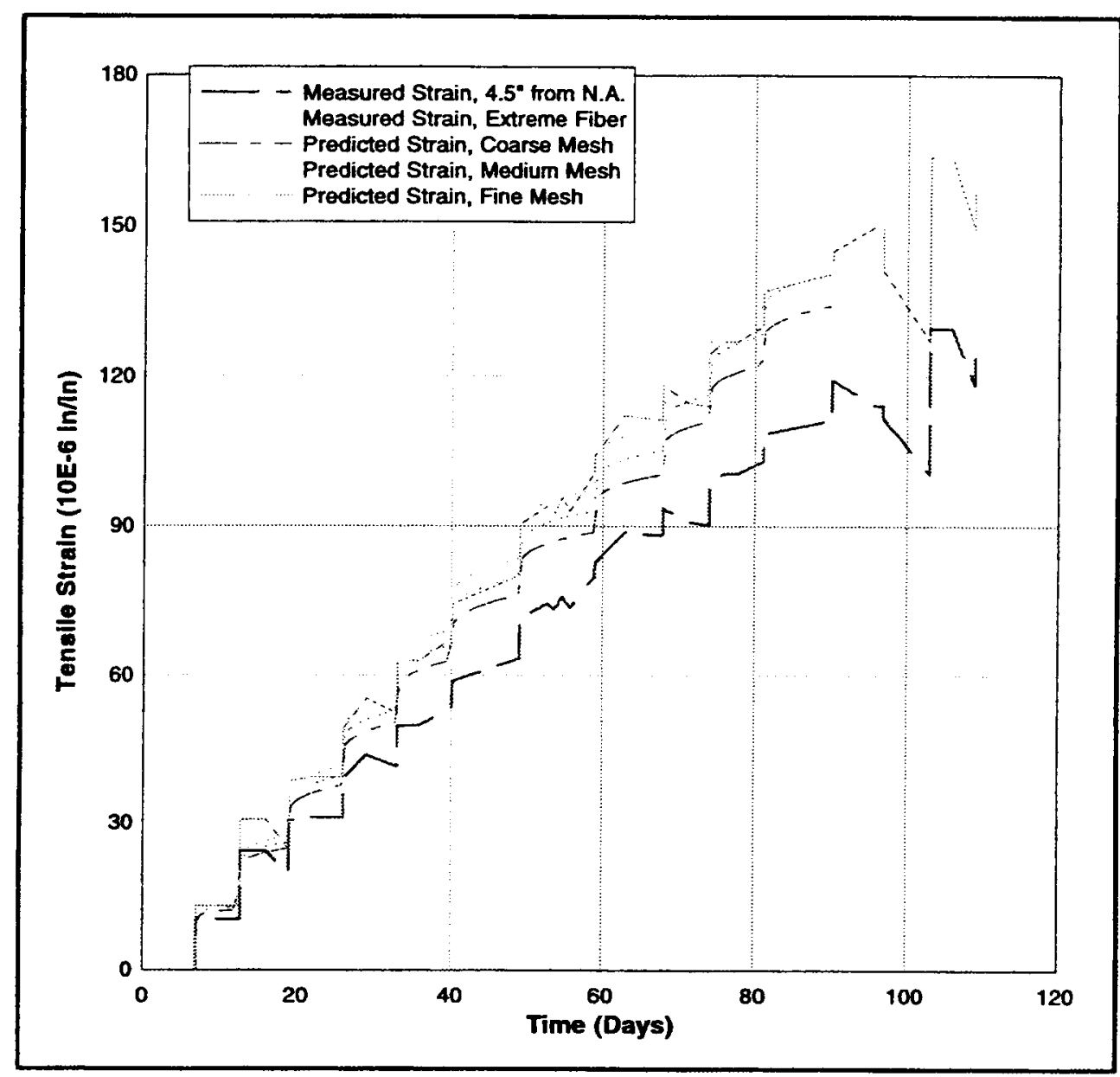

Figure 27. Strain time-histories of measured strains in actual test and predicted strains from numerical analyses using constant value of 117.2 in./in. for $\epsilon_{f}$

while the results from the medium and coarse meshes fall in between the two sets of measured strains. This is due to the location of the integration points in each of the analyses. The bottom integration points of the fine mesh are only 0.423 in. from the base of the beam, while the bottom integration points for the medium mesh and the coarse mesh are 0.634 in. and 0.845 in. from the bottom of the beam, respectively. This puts the location of the integration points for the medium and coarse meshes close to the middle point between the two actual gauges. Figures 27 and 28 also demonstrate the effects of using a common value for $1 / 2 \epsilon_{f}$ as opposed to using an adjusted value. When a single value is used, the analyses (Figure 27) all fail at different times but the strain value at which they fail is approximately the same. However, when the values are adjusted for the mesh (Figure 28), the failure for each analysis occurs on the same day but the strain values at which failure occurs vary.

Questions may be raised concerning the fact that the predicted strains match the actual measured strains so closely, yet the failure times are so different. The discrepancy occurs because the strain used by the constitutive model to predict 


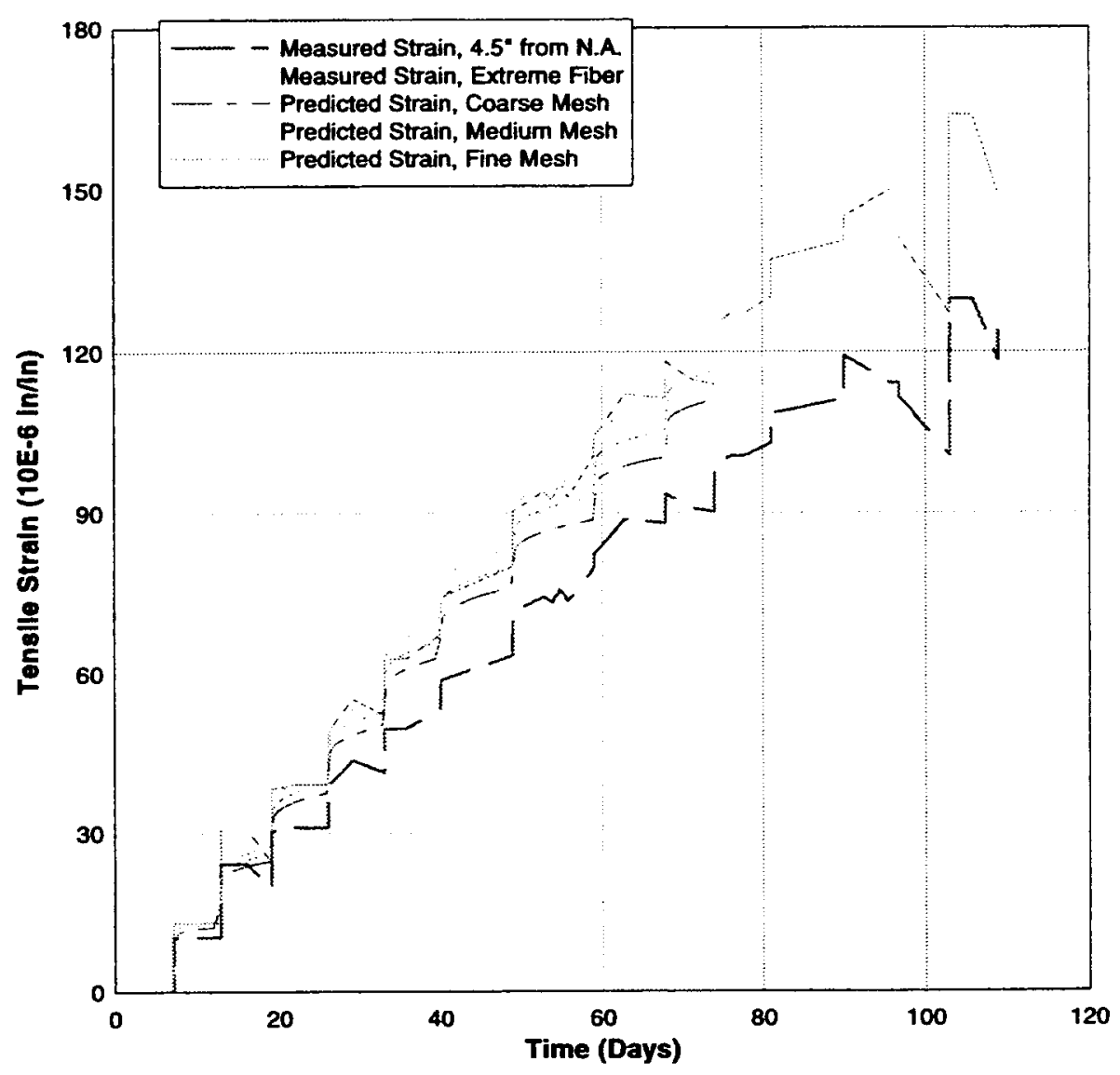

Figure 28. Strain time-histories of measured strains in actual test and predicted strains from numerical analyses using adjusted values for $\epsilon_{\text {, }}$

fracture is different than the total strain that is plotted in Figures 27 and 28 . The strain plotted in these figures includes shrinkage strains due to the autogenous shrinkage which is included in the constitutive model. These strains are not part of the fracture strain used to predict cracking since these strains do not produce a mechanical type of strain which causes fracture. The shrinkage strains can be compared with thermal strains which can create volumetric changes without putting a body under stress if there are no restraining conditions present. Therefore, shrinkage strains associated with autogenous shrinkage will not contribute to the cracking of the beam since, for a simple beam arrangement, there is nothing restraining the movement associated with these strains.

Comparing Figure 28 with Figure 29 shows how the total strain is different from the fracture strain. As can be seen in Figure 29, the strains are higher than those in Figure 28 since the shrinkage strains have been removed.

As can be seen in Figure 29, the fracture strains at failure are approximately the same values as the maximum measured strain before failure at the extreme fiber (second curve in Figure 29). This indicates that the failure strain in the constitutive model accurately reflects the strain state at failure. However, while 


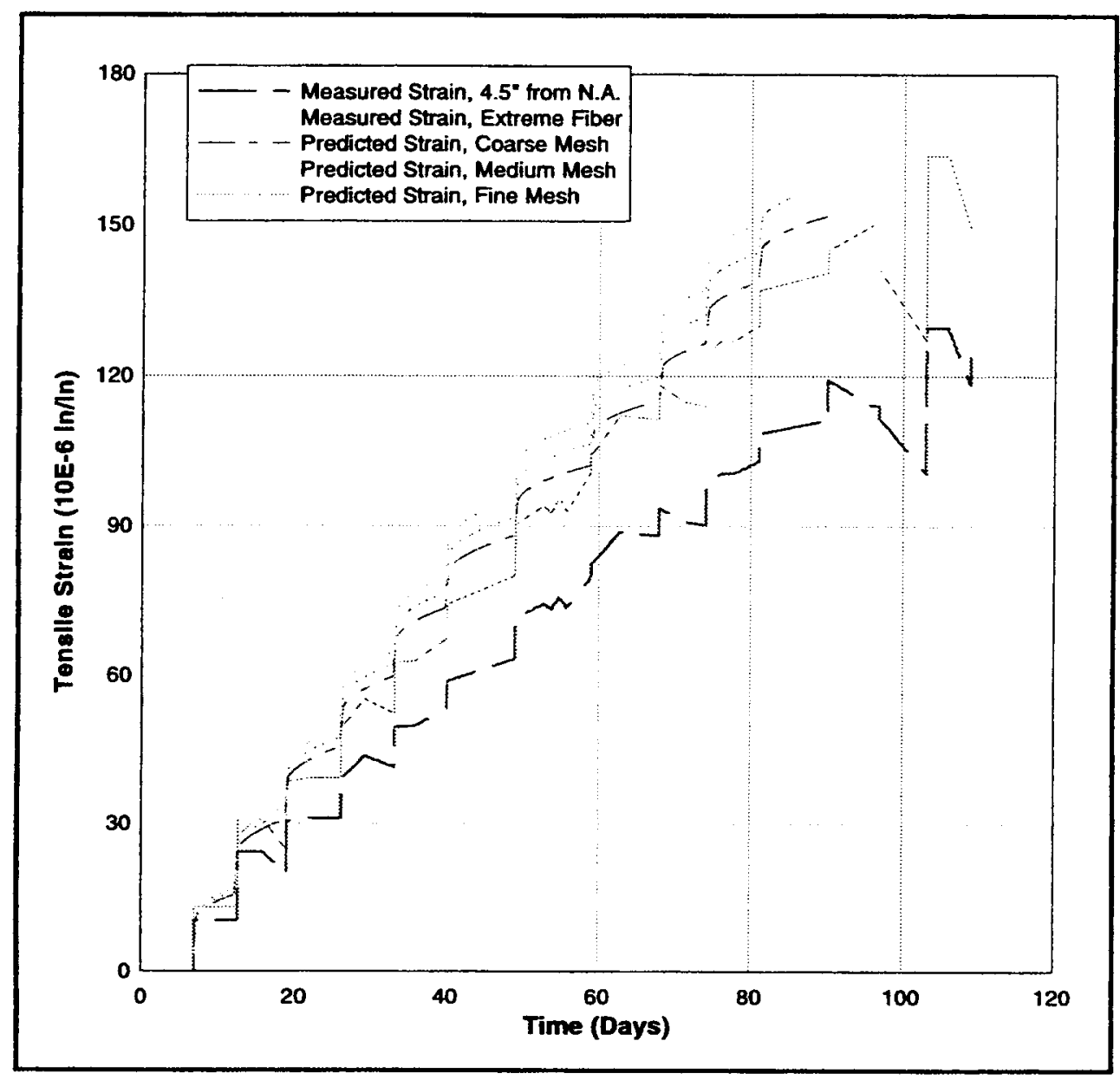

Figure 29. Strain histories of measured strains in actual test and predicted cracking strains from numerical analyses using adjusted values of $\epsilon_{\text {, }}$ (similar to Figure 28) (N.A. = neutral axis)

the strain states may be similar, the stress states are different. The load at which the beam failed in the numerical studies is approximately 30 percent less than the load at which the actual beam failed. This indicates that the strain to which the model is calibrated should be adjusted to remove shrinkage strains prior to performing the calibration in order to capture the correct behavior.

During the study, another factor came into consideration regarding the creep. Currently the constitutive model includes a temperature effect for the creep. This temperature effect was established for high temperature applications such as concrete used in nuclear reactors and for which there were data available. However, the same relationships are used in the constitutive model used in NISA studies, and physical data do not exist to support the use of such a temperature effect in the constitutive model for concrete in the temperature ranges typically observed in mass concrete structures. This temperature effect can be seen when the predicted strain histories shown in Figure 30 are compared with those in Figure 28. The strains shown in Figure 30 are a result of performing analyses in which the initial temperature is not specified in the ABAQUS input deck, and 


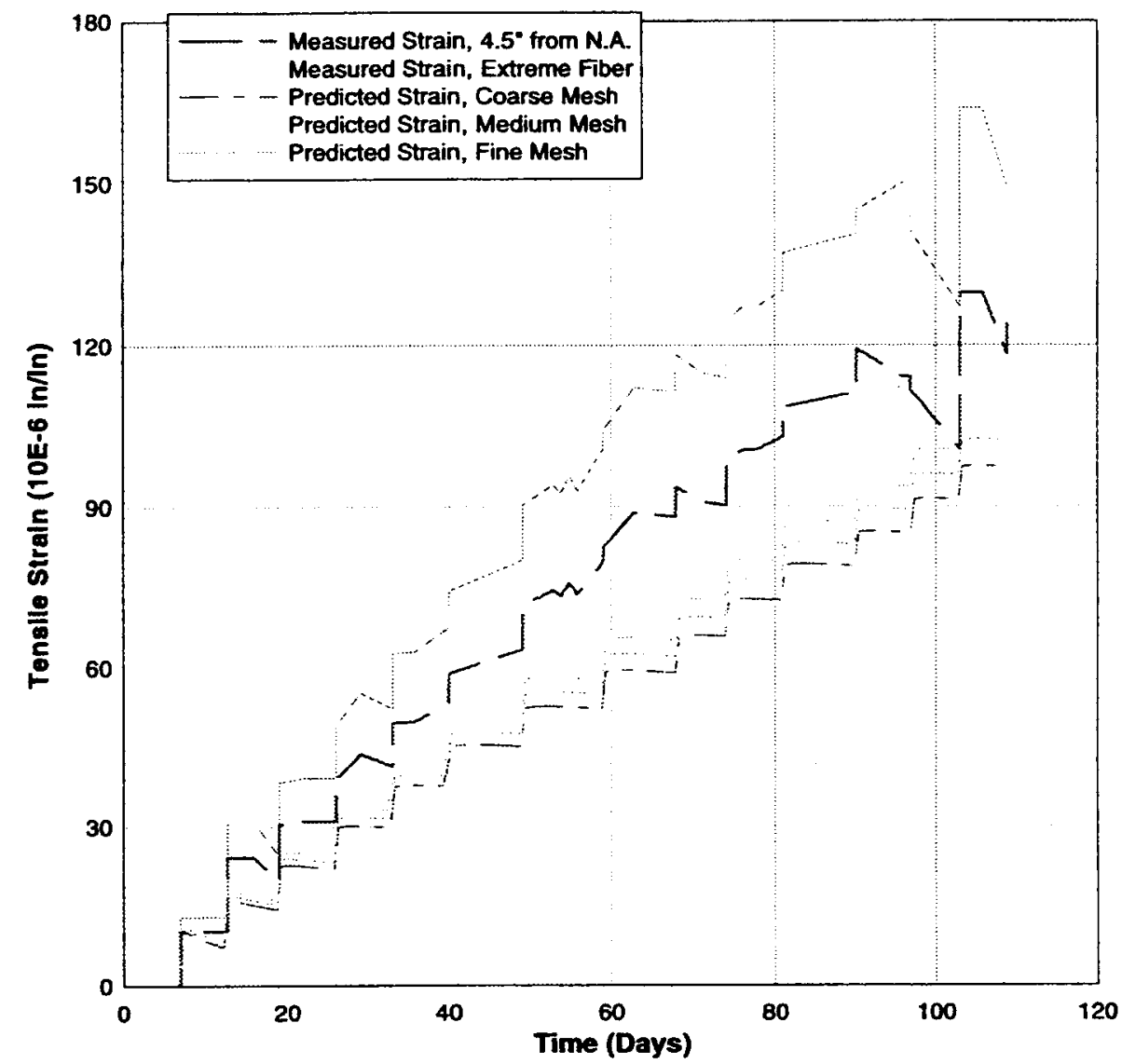

Figure 30. Strain time-histories of measured strains in actual test and predicted strains from numerical analyses using adjusted values for $\epsilon_{f}$ and no initial temperature of $70^{\circ} \mathrm{F}$

therefore a temperature of $0^{\circ} \mathrm{F}$ will be used by ABAQUS to perform the analysis. This $0^{\circ} \mathrm{F}$ assumption will be transferred to the constitutive model and the resulting change is seen in Figure 30 . The original analyses used an initial temperature of $70^{\circ} \mathrm{F}$, for which results are presented in Figure 28, which corresponds to the temperature used to perform the test.

As can be seen in Figure 30, the predicted strains for the case where the temperature is not initialized at $70^{\circ} \mathrm{F}$ are much lower than those predicted when the $70^{\circ} \mathrm{F}$ initial temperature is used. Analyses of all three meshes produced failure at day 109 of the analysis which corresponds to the actual beam failure in the laboratory. While this would seem to indicate that the case using no initial temperature accurately captures the beam behavior, review of the predicted strain histories shows how this case underpredicts the actual strain. That this case matches the same day of failure as the actual tests is at least partly due to the fact that the fracture strain does not include shrinkage strains. Plotting the fracture strains for this case shows that these strains follow more closely the measured strains. This is demonstrated in Figure 31 where the predicted strains (the 


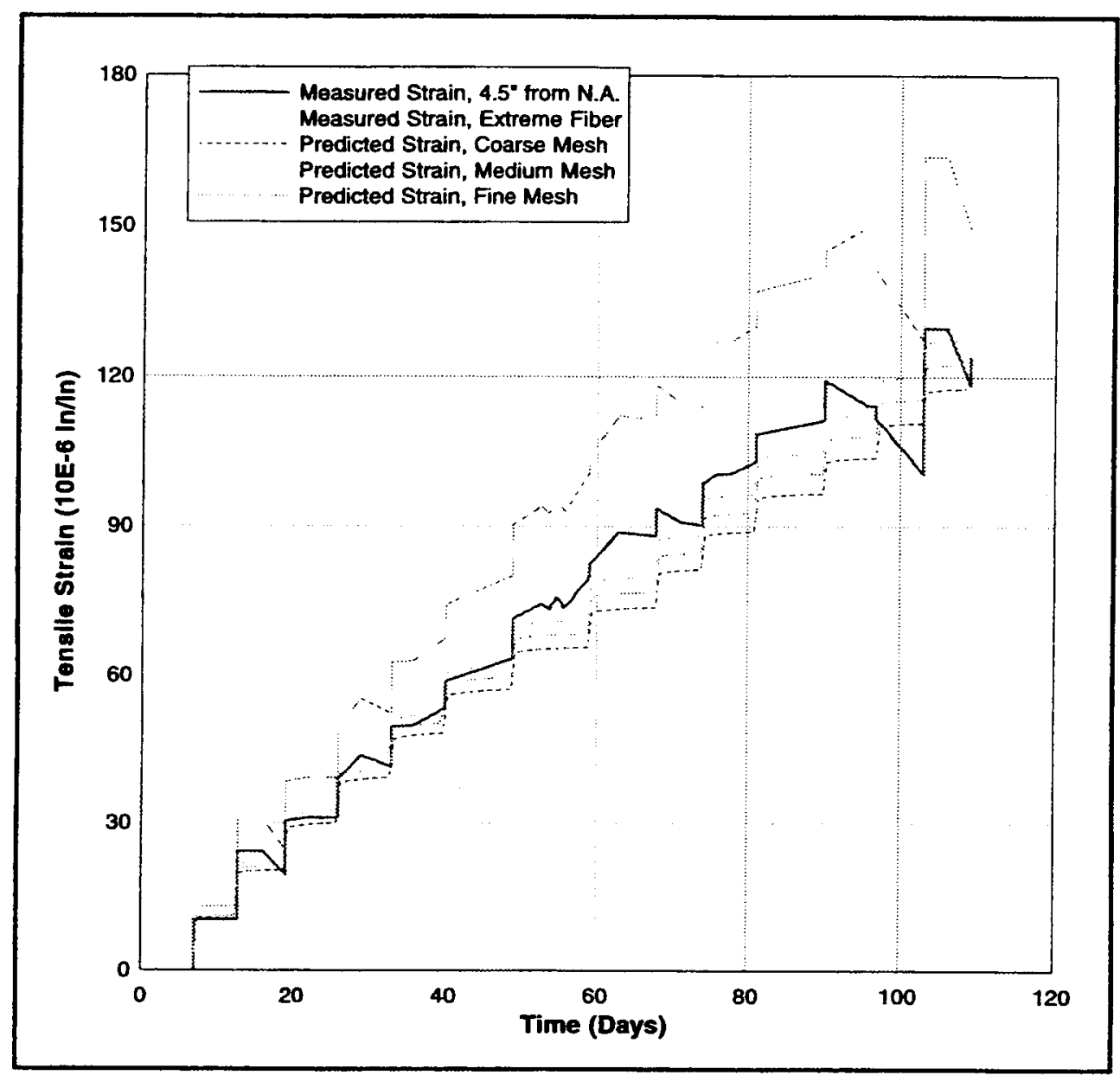

Figure 31. Strain time-histories of measured strains in actual test and predicted strains from numerical analyses using adjusted values for $\epsilon_{f}$ and no initial temperature of $70^{\circ} \mathrm{F}$ but with shrinkage strains included (similar to Figure 30 )

fracture strains) closely approximated the measured strains located 4.5 in. from the neutral axis.

\section{Conclusion}

The predictions made by the numerical analyses of the slow load beam test showed good agreement with the measured data taken during the test. While the comparison of total strains was good, the numerical studies showed that the beam failed much earlier in the loading history than actually occurred. This indicates that the method of calibration must be adjusted. Since it is true that strains in a simple beam due to autogenous shrinkage will not induce cracking, these strains should not be included in the fracture strain used to predict cracking. However, the strain that is being measured in the beam test is the total strain and does include all strains that are occurring in the beam, including any shrinkage strain. 
Therefore, while the shrinkage strain does not contribute to the cracking of the beam, it is still present and must be taken into account when comparing with strains measured.

Strains associated with creep should be considered in a similar manner as the shrinkage strains. Since creep is the elastic deformation of the concrete, it generally will not contribute to cracking of the concrete unless some sort of restraint is introduced. Therefore, the correct method for modeling the strain would be to separate the creep strain from the total strain used to evaluate fracture as is done with the strain associated with autogenous shrinkage. The proprietary owner, ANATECH, of the ANACUP-U software (1992b) which contains the constitutive model, is in the process of updating the model to separate the creep strain from the total strain which should correct the discrepancies observed in the above study. Once this update has occurred, accounting for the creep strain prior to calibration will also need to be considered. In addition, ANATECH currently has plans to remove the temperature dependency of the creep relationship on temperature since there are no data available to support this dependency at temperatures of $100^{\circ} \mathrm{F}$ and less.

Another matter which should be considered in future studies is a possible reduction of the failure strain input into the ABAQUS deck. The computation of the failure strain as currently performed uses stress and strain occurring at the extreme tensile fiber of the beam. It was demonstrated that the coarser the mesh, the longer it takes to actually attain fracture. While this should be intuitively obvious, it does point out that consideration should be given to the fact that the numerical analysis does not perform its calculation at the extreme fiber and that the cracking threshold should be adjusted to account for this discrepancy. 


\section{$5 \quad$ Rapid Load Beam Test}

\section{Introduction}

The rapid load beam test is used to measure the fracture strain in a rapid loading environment to determine strains at fracture without the inclusion of time-dependent factors such as creep and shrinkage. The results of the rapid load beam test are not required for input into the constitutive model, but it will be of value to make comparisons between rapid load beam test data and results predicted in a numerical analysis utilizing the constitutive model.

The setup of the rapid load beam test is the same as the slow load beam test; i.e., it is comprised of a simply supported beam equally loaded at the third points. Figure 22 shows how the test is set up as well as the dimensions used. The beam is loaded through the use of a hydraulic ram operated under electronic control. The loading of the beam is increased continuously at a rapid rate until the beam fractures.

\section{Test}

The test setup shown in Figure 22 is based on ASTM C 78-75 (ASTM 1992b). The test specimen is 12 in. wide. The loading of the beam was based on applicable provisions of C 71-80 (USACE 1949) which specify a loading rate that will increase the stress in the extreme fiber of the beam by 40 psi per minute. Based on the dimensions of the beam used for the testing of the interior mixture for the McAlpine Lock Replacement Project, this loading rate requires that a load of $1,152 \mathrm{lb}$ be applied each minute to the beam. Unlike the slow load beam test, the rapid load beam test is set up for automatic loading. The loading takes place as specified and readings of load and strain are recorded every ten seconds.

Two rapid load beam tests were performed: one when the concrete was 7 days old, and the other at day 109 when the slow load beam failed. The test performed at day 7 failed with a load of 5,509 lb which meant the stress in the extreme tensile fiber was 191.3 psi. For the beam tested at day 109 , the beam failed under a load of $15,414 \mathrm{lbs}$ which induced a stress of $535.2 \mathrm{psi}$. Maximum tensile strains at failure were $63 \mu \mathrm{m}$ for the day 7 test and $119 \mu \mathrm{m}$ at day 109 . 


\section{Numerical Analyses}

As in the slow load beam test, numerical analyses were performed on three different meshes. A coarse, medium, and fine mesh were used and are shown in Figure 26. The coarse mesh used elements that were 4 in. by 4 in., the medium mesh used elements that were 3 in. by 3 in., and the fine mesh used elements that were 2 in. by 2 in. Analyses of the rapid load test were performed at 7 days and at 109 days to match the tests performed in the laboratory. For both analyses, the time history of the beam included the time up until loading. The beam was modeled as being fully supported along the bottom of the beam up until the time of loading for both analyses.

Results of the analyses compared with the actual test data are shown in Figures 32 and 33 in the form of stress-strain plots. The numerical results shown have all been adjusted so that the stresses and strains at the beginning of loading are zero. In addition, the resulting strains reported from the analyses at the integration points have been interpolated so that strains at the extreme fibers are actually plotted. This was done since the strains obtained from the test data had also been adjusted to the extreme fibers. As a result of these adjustments, the curves of the three sets of numerical data all fall on top of one another and give the appearance of only one set of numerical data.

Figure 32 shows the results of the rapid load beam test at an age of 7 days compared with those of the numerical analyses performed. While there is some discrepancy between the two sets of data, the difference is not large. Since the modulus of elasticity can be determined from stress-strain plots, some explanation of the difference may be discovered from computing the predicted modulus based on the curves. Based on the strain values at an applied stress of $17.3 \mathrm{psi}$ and $118.7 \mathrm{psi}$, the modulus for the actual test is approximately $3,343,000 \mathrm{psi}$, while using strains associated with stresses of $20 \mathrm{psi}$ and $240 \mathrm{psi}$ for the numerical studies results in a modulus of approximately $2,371,000 \mathrm{psi}$. The modulus predicted by the numerical study matches well with the modulus of elasticity curve used in the model which has a 7-day modulus of 2,404,000 psi. The numerical results also compare favorably with that reported in Bombich, Neeley, and Garner ${ }^{1}$ which shows a modulus of $2,300,000$ psi at day 7 . From this it is evident that the numerical model is predicting the stress-strain response correctly based on the information for which it was calibrated. While measuring enrors or changes in mixture proportions are possible reasons for the differences seen, they are not likely to occur. A more plausible reason for the discrepancy between the results predicted by the numerical study and the results from the tests can be attributed to the fact that the concrete used in the test to determine the modulus of elasticity is wet-sieved prior to testing while the concrete for the beam test is not. The fact that the concrete used in the beam test is not wet-sieved means that it contains more aggregate. Since the aggregate has a much higher modulus than the concrete at early ages (i.e., 7 days), the presence of more aggregate in a

\footnotetext{
'Op. cit.
} 


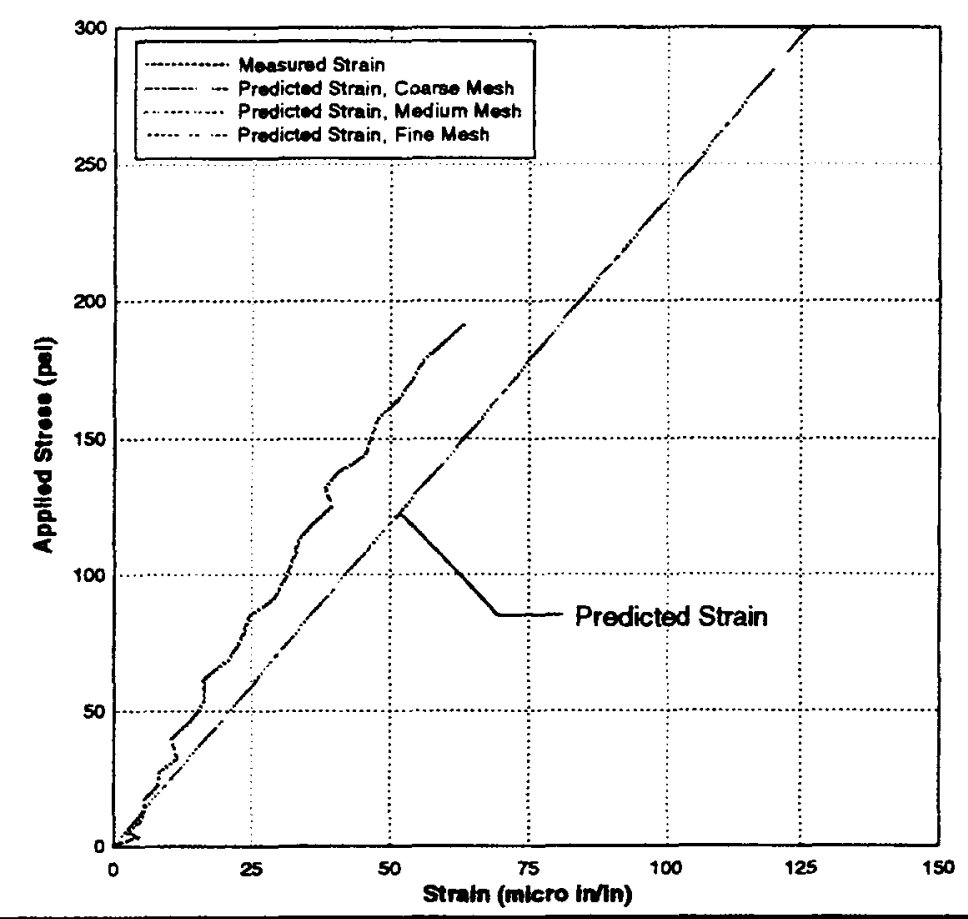

Figure 32. Measured strain versus predicted strain for rapid load test at concrete age of 7 days

concrete mixture will result in a stiffer concrete due to bridging that will occur between aggregate particles.

It should be noted that while the numerical model appears to have performed well in capturing the stress-strain behavior of the concrete, it appears that at early times it may overpredict the cracking capacity of the concrete. The numerical study indicated the strain in the concrete was nearly double of that tested, and the stress was 50 percent greater. Due to the difference in the modulus of the actual beam test and the modulus used in the constitutive model, determining the cause for these differences is difficult. The difference could be a result of the constitutive model being unable to properly capture the behavior of the concrete but this cannot be expressed with certainty unless the constitutive model is calibrated to the concrete mixture actually being used in the test. Therefore, further tests are needed to either confirm or invalidate the observations made in this study.

Figure 33 shows the comparison between the rapid load test performed at day 109 and the numerical studies performed. Comparison of test results with numerical results are very good in this plot. After the initial loading, the two curves look to be almost completely parallel. This is evidenced in the calculation of the modulus of each curve. For the actual test data using strains with associated stresses of $100 \mathrm{psi}$ and $442 \mathrm{psi}$, a moctulus of 4,343,000 is calculated, while the numerical studies strains with associated stresses of $40 \mathrm{psi}$ and $440 \mathrm{psi}$ result in a modulus of $4,326,000$ psi. The calibration of the numerical model shows that at day 109 the modulus of the material is $4,380,000$ psi which 


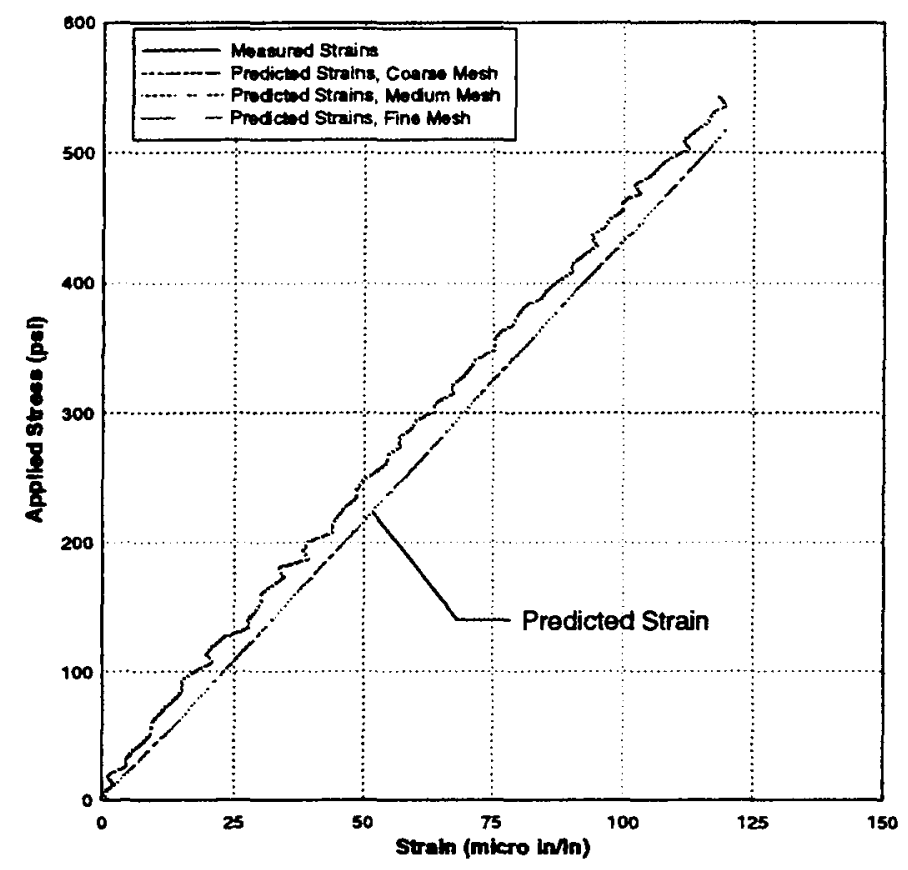

Figure 33. Measured strain versus predicted strain for rapid load test at concrete age of 109 days

compares well with the modulus values computed based on test data and numerical data. There is no actual data for the modulus of elasticity in the materials report ${ }^{1}$ but if an interpolation between the values given at day 90 and day 180 is performed the resulting modulus is $4,180,000$ psi, which once again compares favorably with both sets of data. The similarity of the modulus test and beam test at this point in time can be attributed to the fact that the values of the rock modulus and concrete modulus are much closer at day 109 than at day 7.

Unlike the rapid load test performed at day 7 , the stress and strain values at failure compare very well. The strain at fracture is nearly identical (119 $\mu \mathrm{m})$, and the applied stress is only slightly higher for the experimental results compared with the numerical results. These results indicate that the material model at later times predicts the behavior of the concrete very well, including the load and strain at which it fails.

\section{Conclusions}

The results of the numerical studies of the rapid load beam test compared favorably with the results of the actual experimental results. The differences in the experimental results and numerical results were more than desired, but it is

${ }^{1}$ Op. cit. 
unclear if this is an actual difference or not since the modulus of elasticity predicted in the experimental results does not match the expected modulus for the concrete at an age of 7 days. With respect to the design, the model performed extremely well; i.e., the modulus of elasticity values predicted by the stress-strain curves matched the values to which the model was calibrated. Since questions still remain about the strains at early times, further testing should be performed which will permit some of the questions raised concerning early time strains during rapid loading to be answered. 


\section{Conclusions and Recommendations}

The data contained within this report do not answer all of the questions regarding the validity of the constitutive model used in a NSA, but they do provide insight into where the model does and does not perform well. Overall, the results presented indicate that the constitutive model is capable of approximating results of actual tests. Presented below are specific conclusions which can be made regarding the constitutive model as well as recommendations of what may be needed in the future to assist in validation of the model.

\section{Conclusions}

The analyses performed for comparison with multiaxial creep data compared very well with test data for low levels of compressive loadings but did not perform well for high compressive loads outside of the elastic creep range for which the model was intended. The fact that the model cannot capture the behavior of creep under high compressive loads does not detract from the ability of the model to be used in analyzing massive concrete structures since compressive loads will rarely exceed loadings greater than 40 percent of the compressive strength, which is the basis of the model. It is possible that this limit might be exceeded in dynamic load situations, so caution should be exercised if attempting to use the model for this type of condition. Based on the results of the studies reported in Chapter 2 for loadings under 40 percent of the compressive load, the creep curves which are based on uniaxial tests do provide reasonable predictions for other states of stress.

The analyses for the creep strain recovery were not as successful as the multiaxial creep studies. Results of the analyses did not compare well with the test data. As noted in Chapter 3, the constitutive model was developed under the assumption of a linear creep curve which allows the principle of superposition to be used. However, the principle of superposition does not accurately predict the decrease in strains due to unloading (Bazant 1988). This would explain the large differences between experimental and numerical results observed in Chapter 3 . The cases observed are for instances where the unloading occurs suddenly and 
the creep recovery is overpredicted. For cases in which the unloading occurs gradually, such as in massive concrete structures, the model may be adequate.

Strain comparisons of total strain were very good for the slow load beam test. The strain histories predicted by the three different meshes used in the numerical studies fell between the strain histories recorded by the gauges located at the bottom of the beam and at 1-1/2 in. from the bottom of the beam. This indicates that the numerical predictions were very good since the integration points from which the numerical data were taken are located between the points where the strain gauges on the beam were mounted. While the total strains compared well, the load at which fracture occurred was significantly different. The numerical studies predicted a failure of the beam at approximately 70 percent of the load which caused failure in the laboratory. This difference is attributed to the fact that the constitutive model subtracts strain associated with autogenous shrinkage from total strain. It is true that strains associated with autogenous shrinkage do not contribute to fracture; however, the model is currently calibrated to the total strain as measured during the test. This means that the current method of calibration creates a situation where a model that excludes shrinkage strain as part of the fracture strain is calibrated to a strain that does include shrinkage strain. This is of course inconsistent, and the strains obtained during testing should be adjusted so that the constitutive model can be properly calibrated.

Comparisons made for the rapid load beam test differed for the two times at which it was performed. The test performed at an age of 7 days resulted in a difference in the total strains reported, but due to the fact that the stress-strain curve plotted based on the numerical studies provided a modulus of elasticity value close to that expected for the concrete tested at an age of 7 days, it can be reasoned that wet-sieving the concrete has a definite effect on the modulus of elasticity at early times. At day 109 , when the other rapid load beam test was performed, agreement between the measured results and numerical results was very good and indicated that the model can accurately predict strains in a rapid load situation.

\section{Recommendations}

Based on studies performed on multiaxial creep tests, it appears that the calibration of the creep function as it is currently performed is sufficient for use in NISA studies as they are currently conducted. The only recommendation regarding the creep model is that caution should be exercised in using the current model in a dynamic type analysis since it will not perform well for cases of nonlinear creep and for stress states that are high compared with the compressive strength of the concrete.

For the studies on creep recovery it is clear that a linear definition of creep does not sufficiently capture the strain recovery that occurs when sudden drops in load occur. The data available do not allow an assessment to be made regarding creep strain recovery when the load is reduced gradually. It is possible that the 
model could properly predict creep strain recovery under gradual unloadings as occur in a massive concrete structure, but confirmation of this is needed. It is therefore recommended that at least one series of creep tests be performed which allow a gradual unloading of the specimens and another series which would introduce the unloading in a series of sudden drops in load. Tests such as these would provide the information necessary for determining the accuracy of the constitutive model with respect to capturing creep recovery strain.

The comparisons made of total strains from the numerical studies provided excellent agreement with the laboratory results. However, evaluations of the strains made it apparent that the calibration was not being performed properly. Currently, the fracture strain is calibrated to the measured strain from the laboratory tests, which is the total strain. In its current form, the fracture strain is the total strain reduced by the strain associated with autogenous shrinkage. It is clear that for the fracture strain to be properly calibrated, the strains measured in the laboratory must be reduced by an estimated amount of shrinkage strain prior to performing the calibration. As noted above, a future version of the constitutive model will remove the strains associated with creep as well. It is recommended that in the future, prior to calibrating the fracture strain as outlined in ETL 11102-365 (USACE 1994), the strain at fracture obtained from the slow load beam test be reduced by the amount of strain predicted by the calibrated shrinkage curve in the constitutive model. This will ensure that the comparison is of fracture strain rather than total strain.

While the rapid load beam test comparisons were very good at a concrete age of 109 days, the discrepancy between numerical results and test results at the concrete age of 7 days was inconclusive because the modulus of elasticity calculated from the stress-strain curve for the test results did not match the expected value. It is recommended that an additional beam test be performed at an age of 7 days and that tests for measuring the modulus of elasticity be performed on the same concrete mixture used in the beam tests. This will likely require that larger cylinders than are normally used due to the presence of larger size aggregate because no wet-sieving will be performed. It is also recommended that these tests be performed using the interior concrete mixture from the McAlpine Lock Replacement Project. This will allow confirmation of the ability of the constitutive model to accurately define the behavior due to rapid loads at young ages or to identify an area that may need to be revised in the constitutive model. 


\section{References}

American Concrete Institute. (1970). Designing for effects of creep, shrinkage, and temperature in concrete structures. ACI Publication SP-27, Detroit, MI.

American Society for Testing and Materials. (1992a). "Standard test method for creep of concrete in compression," C 512-87, Philadelphia, PA.

American Society for Testing and Materials. (1992b). "Standard test method for flexural strength of concrete (using simple beam with third-point loading)," C 78-75, Philadelphia, PA.

ANATECH Research Corporation. (1992a). ANACAP-U ANATECH concrete analysis package, version 92-2.2 theory manual. San Diego, CA.

ANATECH Research Corporation. (1992b). ANACAP-U ANATECH concrete analysis package, version 92-2.2 user's manual. San Diego, CA.

Bazant, Z.P. (1988). "Material models for structural analysis." Mathematical modeling of creep and shrinkage in concrete. John Wiley and Sons Ltd., Chichester, England.

Garner, S., Hammons, M. and Bombich, A. (1991). "Red River Waterway thermal studies, Report 2, thermal stress analysis," Technical Report SL-90-8, U.S. Army Engineer Waterways Experiment Station, Vicksburg, MS.

McDonald, J. E. (1973). "Multiaxial creep in concrete," Miscellaneous Paper C-76-6, U.S. Army Engineer Waterways Experiment Station, Vicksburg, MS.

Sandhu, R. S., Wilson, E. L., and Raphael, J. M. (1968). "Two-dimensional stress analysis with incremental construction and creep," Report 67-34, Structural Engineering Laboratory, University of Califomia, Berkeley, CA.

Truman, K. Z., and Fehl, B. D. (1995). "Constitutive modeling of concrete for massive concrete structures, A simplified overview," Technical Report ITL-95-8, U.S. Army Engineer Waterways Experiment Station, Vicksburg, MS. 
U.S. Army Corps of Engineers. (1949). "Standard practice for selecting proportions for normal, heavyweight, and mass concrete (ACI 211.1-81)," C 71-80, Vicksburg, MS.

. (1990). "Special design provisions for massive concrete structures," ETL 1110-2-324, Washington, DC.

(1994). "Nonlinear, incremental structural analysis of massive concrete structures," ETL 1110-2-365, Washington, DC.

Wilson, E. L. (1968). "The determination of temperatures within mass concrete structures," Report 68-17, Structural Engineering Laboratory, University of California, Berkeley, CA.

York, G. P., Kennedy, T. W., and Perry, E. S. (1970). "Experimental investigation of creep in concrete subjected to multiaxial stresses and elevated temperatures," Research Report 2864-2, Department of Civil Engineering, The University of Texas, Austin, TX. 


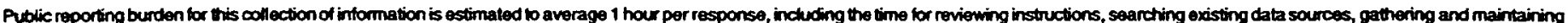

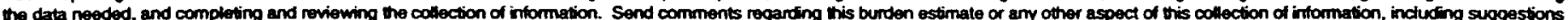

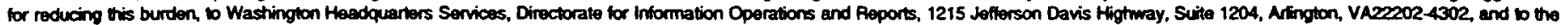
Office of Management and Budget Paperwork Reduction Project (0704-0188), Washington, DC20503.

\begin{tabular}{|l|c|c|}
\hline 1.AGENCY USE ONLY (Leave blank) & $\begin{array}{c}\text { 2.REPORT DATE } \\
\text { June } 1998\end{array}$ & $\begin{array}{c}\text { 3.REPORT TYPE AND DATES COVERED } \\
\text { Final report }\end{array}$
\end{tabular}

\begin{tabular}{|l|l|}
\hline $\begin{array}{l}\text { 4.TITLE AND SUBTTLE } \\
\text { Investigation of the Constitutive Model Used in Nonlinear, Incremental } \\
\text { Structural Analyses }\end{array}$ & 5.FUNDING NUMBERS \\
\hline $\begin{array}{l}\text { 6.AUTHOR(S) } \\
\text { Barry D. Fehl, Sharon B. Gamer }\end{array}$ & $\begin{array}{l}\text { 8.PERFORMING ORGANIZATION } \\
\text { REPORT NUMBER } \\
\text { Technical Report ITL-98-1 }\end{array}$ \\
\hline $\begin{array}{l}\text { 7.PERFORMING ORGANIZATION NAME(S) AND ADDRESS(ES) } \\
\text { 3909 Halls Ferry Road, Vicksburg, MS 39180-6199 }\end{array}$ & $\begin{array}{l}\text { 10.SPONSORINGMONITORING } \\
\text { AGENCY REPORT NUMBER }\end{array}$ \\
\hline $\begin{array}{l}\text { 9.SPONSORINGMONITORING AGENCY NAME(S) AND ADDRESS(ES) } \\
\text { WaShington, DC 20314-1000 }\end{array}$ & \\
\hline
\end{tabular}

\section{SUPPLEMENTARY NOTES}

Available from National Technical Information Service, 5285 Port Royal Road, Springfield, VA 22161.

12a.DISTRIBUTIONAVALABILTY STATEMENT

Approved for public release; distribution is unlimited.

13.ABSTRACT (Maximum 200 wonds)

Nonlinear, incremental structural analysis (NISA) has been used on Corps of Engineer projects for the last decade. An integral part of the NISA methodology is the constitutive model used when performing a NISA study. This constitutive model contains age-dependent characteristics for the modulus of elasticity, autogenous shrinkage, and creep as well as a stress-strain smeared cracking relationship.

Before performing a NISA study on a project, the constitutive model is typically calibrated to laboratory tests. Despite the fact that the model is calibrated against these tests, full validation of the model requires additional comparisons to data used from past NISA studies as well as data from other sources and finally a full scale test to determine if laboratory tests capture the behavior occurring in a massive concrete structure.

The report contains comparisons made as part of the validation effort. Data obtained from the literature on multi-axial creep states and on creep recovery were used to evaluate the creep relationships in the constitutive model for these conditions. Comparisons are also presented between test data from a Corps of Engineers project and the data predicted by the constitutive model with respect to the smeared cracking aspect of the constitutive model.

(Continued)

\section{SUBJECT TERMS}

Concrete testing

Constitutive model

Creep
Finite elements

Fracture strain

Fracture stress
Massive concrete

Nonlinear, incremental structural analysis Shrinkage
15.NUMBER OF PAGES

54

16.PRICE CODE

20.LIMITATION OF ABSTRACT

\begin{tabular}{|c|c|c|}
\hline $\begin{array}{c}\text { 17.SECURITY CLASSIFICATION } \\
\text { OF REPORT }\end{array}$ & $\begin{array}{c}\text { 18.SECURITY CLASSIFICATION } \\
\text { OF THIS PAGE } \\
\text { UNCLASSIFIED }\end{array}$ & $\begin{array}{c}\text { 19.SECURITY CLASSIFICATION } \\
\text { OF ABSTRACT }\end{array}$ \\
\hline
\end{tabular}

NSN 7540-01-280-5500
Standard Form 298 (Rev. 2-89)

Prescribed by ANSI Sid. Z39-18 298-102 
13. (Concluded).

Conclusions regarding the behavior of the constitutive model are drawn, and recommendations on how to use the model are made. Revisions to the model itself are recommended as well. 